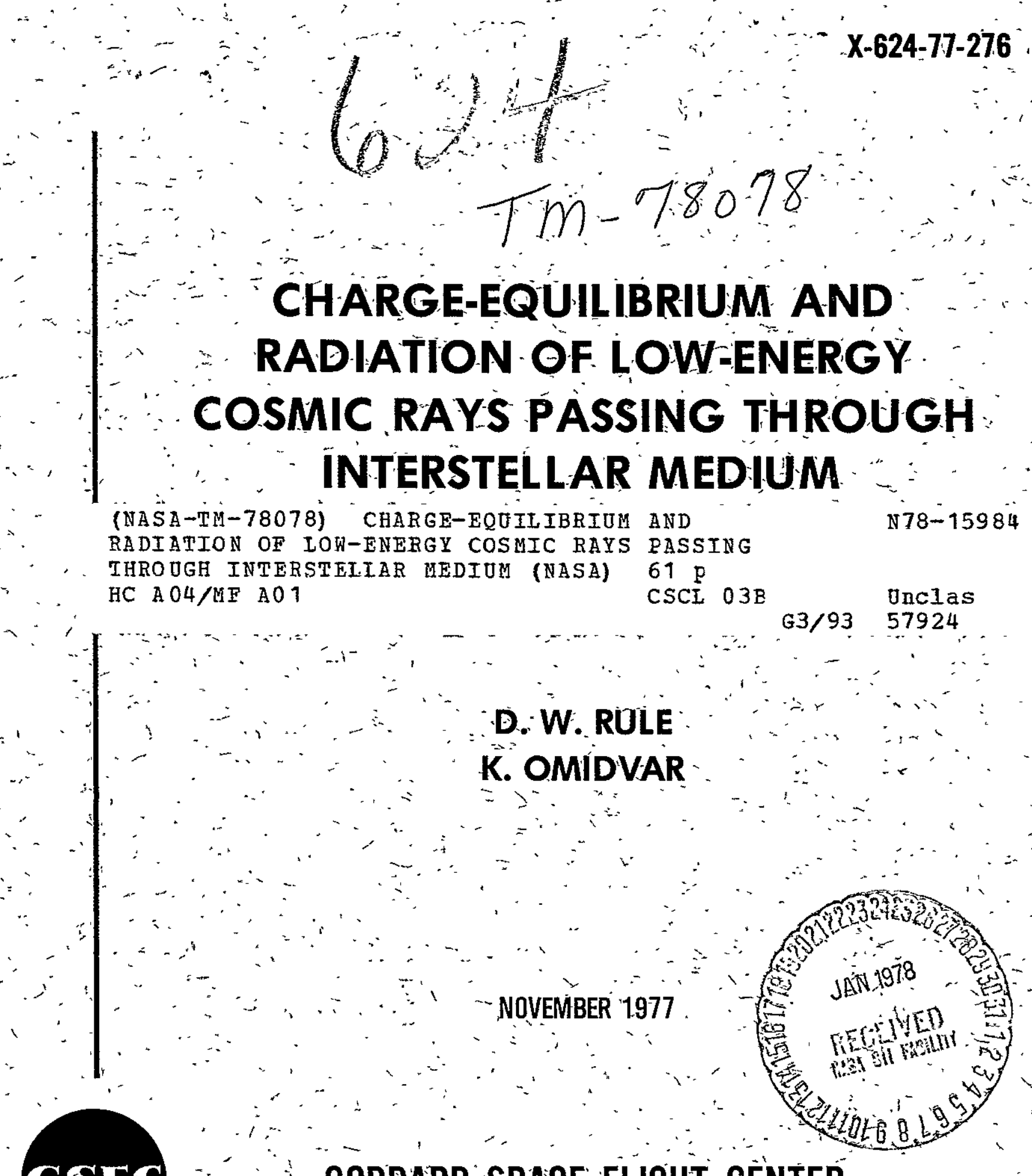

GSFC

GODDARD SPACE FLIGHT CENTER

GREENBELT; MARYLAND 



\title{
CHARGE-EQUILIBRIUM AND RADIATION OF LOW-ENERGY \\ COSMIC RAYS PASSING THROUGH INTERSTELLAR MEDIUM
}

\author{
D. W. Rule \\ K. Omidvar
}

November 1977

GODDARD SPACE FLIGHT CENTER

Greenbelt, Maryland 
ABSTRACT ........................... ii

I. INTRODUCTION ...............

II. CAlCUlation OF CROSS SECtions . . . . . . . . 3
a) Ionization Cross Sections . . . . . . . . . . . 3
b) Excitation Cross Sections . . . . . . . . . . . . 7
c) Charge-Exchange Cross Sections . . . . . . . . . 11

III. CHARGE EQUILIBRIUM AND RADIATION . . . . . . . . . 14

a) Charge Fractions . . . . . . . . . . . . . 14

b) Effective X-Ray Production Cross Sections . . . . . . . 16

c) Multiplicities . . . . . . . . . . . . . 20

IV. DISCUSSION . . . . . . . . . . . . . . . . 22

V. CONCLUSTON . . . . . . . . . . . . . 26

REFERENCES . . . . . . . . . . . . . . . . . . . 28

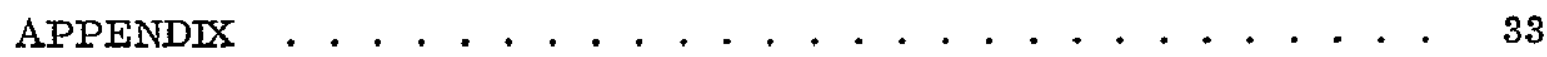

\section{TABLES}

1 Comparison of some experimental and calculated cross sections and their ratios for a number of charge-exchange reactions ................... 31

2 Number of X-Rays per Cosmic Ray Nucleus . . . . . . 32

3 The Coefficients $A_{n}$ and $B_{n}$ of eq. (A1)......... 36 


\title{
CHARGE-EQUILIBRIUM AND RADIATION OF LOW-ENERGY \\ COSMIC RAYS PASSING THROUGH INTERSTELLAR MEDIUM
}

\author{
D. W. Rule* and K. Omidvar \\ Stratosphere Physics and Chemistry Branch \\ Laboratory for Planetary Atmospheres \\ NASA/Goddard Space Flight Center
}

\begin{abstract}
The charge equilibrium and radiation of an oxygen and an iron beam in the $\mathrm{MeV}$ per nucleon energy range, representing. a typical beam of low-energy cosmic rays passing through the interstellar medium, is considered. Electron loss of the beam has been taken into account by means of the first Born approximation allowing for the target atom to remain unexcited, or to be excited to all possible states. Electron capture cross sections have been calculated by means of the scaled Oppenheimer-Brinkman-Kramers approximation, taking into account all atomic shells of the target atoms. Radiation of the beam due to electron capture into the excited states of the ion, collisional excitation and collisional innershell ionization of the ions has been considered. Effective X-ray production cross sections and multiplicities for the most energetic $\mathrm{X}$-ray lines emitted by the $\mathrm{Fe}$ and $\mathrm{O}$ beams have been calculated. Subject headings: atomic and molecular processes - cosmic rays interstellar matter - X-rays.
\end{abstract}

*National Research Council Research Associate 


\section{CHARGE-EQUILIBRIUM AND RADIATION OF LOW-ENERGY \\ COSMIC RAYS PASSING THROUGH INTERSTELEAR MEDIUM}

\section{INTRODUCTION}

When a neutral or charged beam of particles passes through a gaseous medium, through collisions, it reaches a state of charge equilibrium independent of the initial charge state of the beam. The equition rium is reached through the competing processes of ionization of the beam by the surrounding gas, and electron capture by the beam from the gas, the equilibrium time being of the order of the collision time.

Through the collisional excitation of its particles with the surrounding gas the beam radiates. The radiation is in the visible to the $\mathrm{X}$-ray range, depending on the composition of the beam, is Doppler shifted in frequency if the beam is unidirectional, and is Doppler broadened if the beam has an isotropic distribution.

In a number of problems of interest such as the propagation of isotropic low energy cosmic rays through the interstellar medium, or neutral beam injection in a fusion plasma, information about the charge state equilibrium of a passing beam and its radiation distribution and intensity is desirable. This information requires an accurate knowledge of cross sections for charge-exchange, ionization, and excitation processes.

There have been several calculations of charge equilibrium and X-ray production by oxygen and iron ions, e.g., Serlemitsos 1973; Watson 1975; Pravdo 
and Boldt 1975; and Bussard, et al. 1978. However the previous work contains a variety of approximations for the charge exchange, ionization, and excitation cross sections needed as input, and consequently the results of these calculations are not in agreement in several instances. Recently the reliability of the iron ion calculations using scaled Brinkman-Kramers charge exchange cross sections has been questioned (Belkic and McCarroll 1977). For these reasons, the present work focuses on the method of obtaining the input cross sections, all of which have been consistently calculated within the Born approximation. By careful comparison with experimental data wherever possible, we have attempted to assess the reliability of the methods we have used. In this way we have been able to understand the sources of some of the disagreement among previous work. Furthermore, our analysis should provide a reliable'starting point from which to approach similar calculations involving different atomic species.

In the next section a brief description of the theory will be given. In the third and fourth sections results and discussions will be presented. 


\section{CALCULATION OF CROSS SECTIONS}

a) Ionization Cross Sections

The ionization cross sections used in our determination of the equilibrium distribution of charge states were obtained using the first Born approximation (FBA). These calculations are an outgrowth of the methods developed for hydrogen and helium projectiles by Bates and several co-workers (for a review see Bates 1962). Similar calculations of electron loss by hydrogen-like and helium-1ike ions in neutral hydrogen and helium have been done by Dmitriev, et al. (1965) and Senashenko, et al. (1968). Recently, this approach was extended to the calculation of ionization from multi-electron atoms in collision with neutral atoms by Rule (1977). In this section we will simply outline the calculational method. The references given in this paragraph contain more detailed điscussions.

We begin by assuming that the major contribution to electron loss by an ion is direct Coulomb ionization of a single electron. We thus neglect Auger processes, multiple electron ionization, and transfer of electrons from the projectile to any target ions that may be present. We then write the total ionization cross section $\sigma^{\mathrm{I}}$ as a sum over partial cross sections for each occupied subshell of the projectile ion:

$$
\sigma^{I}(V)=\sum_{n=1}^{N} \sum_{\ell} \sigma_{n, \ell}^{I}(V)
$$


where $\mathrm{N}$ denotes the principle quantum number of the highest occupied shell, and $\mathrm{V}$ is the relative velocity of the ion-atom system. Each $\sigma_{\mathrm{n}, \ell}^{\mathrm{I}}$ can be separated into a part which represents ionization processes in which the target is elastically scattered, $\sigma_{\mathrm{e}}^{\mathrm{I}}$, and a part which represents the sum over all ionizations of the projectile ion during which the target atom is inelastically scattered, $\sigma_{i}^{I}$, as

$$
\sigma_{\mathrm{n}, \ell}^{\mathrm{I}}(\mathrm{V}) \equiv \sigma_{\mathrm{e}}^{\mathrm{I}}(\mathrm{V})+\sigma_{\overline{\mathrm{i}}}^{\mathrm{I}}(\mathrm{V})
$$

The FBA expression for these cross sections is (see, e.g., Bates 1962):

$$
\sigma_{\mathrm{e}}^{\mathrm{I}}(\mathrm{V})=8 \pi \mathrm{a}_{0}^{2} \frac{\mathrm{Z}_{\mathrm{T}}^{2}}{\left(\mathrm{Z}_{\left.\mathrm{n}, \mathrm{\ell}^{\mathrm{V}}\right)^{2}}^{2}\right.} \int_{\mathrm{Q}_{1}}^{\mathrm{Q}_{2}} \frac{\mathrm{dQ}}{\mathrm{Q}^{3}}\left|\mathrm{l}-\mathrm{F}\left(\mathrm{Z}_{\mathrm{n}, \ell} \mathrm{Q}\right)\right|^{2} \int_{\mathrm{o}}^{\mathrm{k}_{1}} \mathrm{k}^{2} \mathrm{dk} \varepsilon_{\mathrm{n}, \ell}(\mathrm{k}, \mathrm{Q})
$$

and

$$
\sigma_{\mathrm{i}}^{\mathrm{I}}(\mathrm{V})=8 \pi \mathrm{a}_{0}^{2} \frac{\mathrm{Z}_{\mathrm{T}}}{\left(\mathrm{Z}_{\left.\mathrm{n}, \ell^{\mathrm{V}}\right)^{2}}^{2}\right.} \int_{\mathrm{Q}_{1}^{\prime}}^{\mathrm{Q}_{2}^{\prime}} \frac{\mathrm{dQ}}{\mathrm{Q}^{3}} \mathrm{~S}\left(\mathrm{Z}_{\mathrm{n}, \ell} \mathrm{Q}\right) \int_{\mathrm{o}}^{\mathrm{k}_{1}^{\prime}} \mathrm{k}^{2} \mathrm{dk} \varepsilon_{\mathrm{n}, \ell}(\mathrm{k}, \mathrm{Q})
$$

In the above expressions, $a_{0}$ is the Bohr radius, $Z_{n, l}$ is the effective charge for an electron in the $(n, \ell)$ subshell of the projectile, and

$$
\mathrm{v} \equiv \mathrm{V} / \mathrm{Z}_{\mathrm{n}, \ell^{\mathrm{v}}}
$$

where $v_{0}$ is the Bohr velocity. We also have $\mathrm{Z}_{\mathrm{T}}$, the target's nuclear charge, and $\vec{Q}$, which is equal to $Z_{n, \ell}^{-1}$ times the momentum transfer,

$$
\overrightarrow{\mathrm{Q}} \equiv Z_{\mathrm{n}, \ell}^{-1}\left(\overrightarrow{\mathrm{K}}_{\mathrm{f}}-\overrightarrow{\mathrm{K}}_{\mathrm{i}}\right)
$$

Here $\vec{K}_{\mathbf{i}}$ and $\overrightarrow{\mathrm{K}}_{\mathrm{f}}$ are the initial and final momenta in the center of mass of the projectile-target system in units of $a_{0}^{-1}$. 
The functions in the integrands above are $F(q)$, the target elastic form factor for momentum transfer $\vec{q}$, and $S(q)$, the incoherent scattering form factor for the target atom. The form factor for transition from the $(n, \ell)$ subshell to the continuum state with momentum of magnitude $\mathrm{k}$ is given by

$$
\&_{n, \ell}(k, Q) \equiv \frac{N_{n, \ell}}{2 \ell+1} \int \mathrm{d} \hat{\mathrm{k}} \sum_{\mathrm{m}=0}^{\ell}\left(2-\delta_{\mathrm{m}, \mathrm{o}}\right)\left|\left\langle\overrightarrow{\mathrm{k}}\left|\mathrm{e}^{\mathrm{i} \overrightarrow{\mathrm{Q}} \cdot \overrightarrow{\mathrm{r}}}\right| \mathrm{n} \ell \mathrm{m}\right\rangle\right|^{2}
$$

where $N_{n, l}$ is the number of electrons in the subshell and $|\vec{k}\rangle$ and $|n \ell m\rangle$ are hydrogenic continuum and bound state wave functions. The explicit form for $\varepsilon_{n, \ell}(k, Q)$ is given by Omidvar, et al. (1972).

The limits in Equations (4) and (5) are as follows:

$$
\begin{gathered}
\mathrm{Q}_{1}=\mathrm{I}_{\mathrm{n}, \ell} /\left(2 \mathrm{Z}_{\mathrm{n}, \ell}^{2} \mathrm{v}\right) \\
\mathrm{Q}_{2}, \mathrm{Q}_{2}^{\prime} \approx 2 \mathrm{Z}_{\mathrm{n}, \ell}^{-1} \mathrm{~K}_{\mathrm{i}} \rightarrow \infty,
\end{gathered}
$$

and

$$
\mathrm{Q}_{1}^{\prime} \approx\left(\mathrm{I}_{\mathrm{n}, \ell}+\mathrm{I}_{\mathrm{T}}\right) /\left(2 \mathrm{Z}_{\mathrm{n}, \ell}^{2} \mathrm{v}\right)
$$

The upper limits of the $\overrightarrow{\mathrm{k}}$ integrations are given by

$$
\mathrm{k}_{1}=\left[\frac{\mathrm{m}_{\mathrm{e}}}{\mu}\left(2 \mathrm{QK}_{\mathrm{i}}-\mathrm{Q}^{2}\right)-\mathrm{I}_{\mathrm{n}, \ell}\right]^{\mathrm{I} / 2},
$$

and

$$
\mathrm{k}_{1}^{\prime} \approx\left[\frac{\mathrm{m}_{\mathrm{e}}}{2 \mu}\left(2 \mathrm{QK}_{\mathrm{i}}-\mathrm{Q}^{2}\right)-\frac{1}{2}\left(\mathrm{I}_{\mathrm{n}, \ell}+\mathrm{I}_{\mathrm{T}}\right)\right]^{1 / 2}
$$

where $m_{e}$ is the mass of an electron, and $\mu$ is the reduced mass of projectiletarget system. Also, $I_{n, l}$ is the ionization potential for an electron in the ( $\mathrm{n}, l$ ) 
subshell in Rydbergs and $I_{T}$ is the ionization potential for the neutral target atom. Note that when $\mathrm{v} \gg 1$, the proportionalities

$$
\sigma_{\mathrm{e}}^{\mathrm{I}}(\mathrm{v}) \propto \mathrm{Z}_{\mathrm{T}}^{2} /\left(\mathrm{Z}_{\mathrm{n}, \ell}^{2} \mathrm{v}\right)^{2} \text { and } \sigma_{\mathrm{i}}^{\mathrm{I}}(\mathrm{v}) \propto \mathrm{Z}_{\mathrm{T}} /\left(\mathrm{Z}_{\mathrm{n}, \ell}^{2} \mathrm{v}\right)^{2}
$$

give scaling with respect to $Z_{n, \ell}$ and $v$, which is identical for both $\sigma_{\mathrm{e}}^{\mathrm{I}}$ and $\sigma_{\mathrm{i}}^{\mathrm{I}}$, while the scaling with $\mathrm{Z}_{\mathrm{T}}$ differs in the two cases.

Ionization cross sections for $\mathrm{Fe}^{+25}-\mathrm{Fe}^{+20}$ in hydrogen are shown in Figure 1. The solid curves were calculated as described above, while the dashed curves were obtained from the binary encounter approximation (BEA) as tabulated by Hanson (1973) for ionization by protons. The data of Berkner, et al. (1977) is shown for comparison. Since the original data was taken for Fe-ions in $\mathrm{H}_{2}$, we have divided it by two before plotting. We see that the agreement of the present calculation with the data is quite good. For the case of $\mathrm{Fe}^{+23}$ and $\mathrm{Fe}^{+22}$, the data falls between the BEA and the FBA values; however only a single data point is available for these cases.

We have found that at energies near the peak of the cross sections, the contributions of $\sigma_{\mathrm{e}}^{\mathrm{I}}$ and $\sigma_{\mathrm{i}}^{\mathrm{I}}$ to $\sigma_{\mathfrak{n}, \ell}^{\mathrm{I}}$, Equation (2), are comparable. For example, at $\sim 25 \mathrm{MeV} /$ nucleon $\mathrm{R} \equiv \sigma_{\mathrm{i}}^{\mathrm{I}} / \sigma_{\mathrm{e}}^{\mathrm{I}} \approx 0.6$. At energies lower than that of the peak, $\mathrm{R}$ becomes smaller, e.g., at $0.67 \mathrm{MeV} /$ nucleon $\mathrm{R} \approx 0.5$. The contribution of $\sigma_{\mathrm{e}}^{\mathrm{I}}$ is roughly equivalent to the cross section for ionization of $\mathrm{Fe}$-ions by a proton (see Rule 1977) as was used by Watson (1975), thus neglect of the structure of the hydrogen target atom during the ionizing collision can cause a substantial 
error in the calculated cross sections. For lower charge states of Fe, $\mathrm{Fe}^{+24}-$ $\mathrm{Fe}^{+16}$, the ratio $\sigma_{\mathrm{i}}^{\mathrm{I}} / \sigma_{\mathrm{e}}^{\mathrm{I}}$ is $\sim 0.5$ near the peak of the cross sections. The ionization cross sections for the ions $\mathrm{Fe}^{+19}-\mathrm{Fe}^{+16}$ have also been calculated in the same manner as those in Figure 1 using the FBA.

In Figure 2 the calculated ionization cross sections for single electron loss by oxygen ions are given. The FBA results are again compared to the BEA results. We are not aware of any data for comparison with these calculated values; however, for the ionization of $\mathrm{O}^{+7}-\mathrm{Q}^{+3}$ by He, a detailed comparison of the FBA and BEA values with the data of Macdonald and Martin (1971) has been given by Rule (1977). There it was shown that FBA results were in reasonable agreement with the data for all five charge states considered, while the BEA values over-estimated the cross section considerably for the $\mathrm{O}^{+5}-\mathrm{O}^{+3}$ cases and underestimated them for $\mathrm{O}^{+7}$ and $\mathrm{O}^{+6}$. The relative contributions to $\sigma_{\mathrm{n}, \ell}^{\mathrm{I}}$ from $\sigma_{\mathrm{e}}^{\mathrm{I}}$ and $\sigma_{\mathrm{i}}^{\mathrm{I}}$ of Equation (2) are again indicated by the ratio $\mathrm{R} \equiv \sigma_{\mathrm{i}}^{\mathrm{I}} / \sigma_{\mathrm{e}^{*}}^{\mathrm{I}}$, For $\mathrm{O}^{+7}-$ $\mathrm{O}^{+3}$ in $\mathrm{H}, \mathrm{R} \approx 0.5$ near the peaks of these cross sections, while for $\mathrm{O}^{+7}-\mathrm{O}^{+3}$ in He $\mathbf{R} \approx 0.3$ for peak values. Thus inelastic target processes collectively contribute substantially to the total ionization cross sections for oxygen ions as well as for iron ions.

\section{b) Excitation Cross Sections}

The first Born approximation approach was also used to calculate the excitation cross sections necessary to obtain effective $\mathrm{X}$-ray production cross 
sections. The method is quite similar to that outlined above for the case of ionization. The calculational technique has been described thoroughly by Bates (1962) and also by Levy (1969). We will simply give the results here.

As for ionization, we can divide the total excitation cross section $\sigma^{\mathrm{E}}$ into two parts: $\sigma \sigma_{\mathrm{e}}^{\mathrm{E}}$ and $\sigma_{\mathrm{i}}^{\mathrm{E}}$, which represent excitation of the projectile during elastic and inelastic scattering of the target, respectively. Just as in Equations (3) and (4) we can write:

$$
\sigma_{\mathrm{e}}^{\mathrm{E}}(\mathrm{v})=8 \pi \mathrm{a}_{0}^{2}\left[\frac{\mathrm{Z}_{\mathrm{T}}}{\mathrm{Z}_{\mathrm{n}, \ell^{\mathrm{V}}}^{2}}\right]^{2} \int_{\mathrm{Q}_{1}}^{\mathrm{Q}_{2}}\left|1-\mathrm{F}\left(\mathrm{Z}_{\mathrm{n}, \ell} \mathrm{Q}\right)\right|^{2} \&_{\mathrm{n} \ell, \mathrm{n}^{\prime} \ell^{\prime}(\mathrm{Q})} \frac{\mathrm{dQ}}{\mathrm{Q}^{3}},
$$

and

$$
\sigma_{\mathrm{i}}^{\mathrm{E}}(\mathrm{v})=8 \pi \mathrm{a}_{0}^{2} \frac{\mathrm{Z}_{\mathrm{T}}}{\left(\mathrm{Z}_{\mathrm{n}, \ell}^{2} \mathrm{v}\right)^{2}} \int_{\mathrm{Q}_{1}^{\prime}}^{\mathrm{Q}_{2}^{\prime}} \frac{\mathrm{dQ}}{\mathrm{Q}^{3}} \mathrm{~S}\left(\mathrm{Z}_{\mathrm{n}, \ell} \mathrm{Q}\right) \&_{\mathrm{n} \ell, \mathrm{n}^{\prime} \ell^{\prime}(\mathrm{Q})}
$$

The function $\&_{\mathrm{n} \ell, \mathrm{n}^{\prime} \ell^{\prime}}(\mathrm{Q})$ is defined in a manner analogous to $\&_{\mathrm{n}, \ell}(\mathrm{k}, \mathrm{Q})$ of Equation (7):

$$
\mathbb{E}_{\mathrm{n}^{\prime} \ell, \mathrm{n}^{\prime} \ell^{\prime}}(\mathrm{Q}) \equiv \frac{\mathrm{N}_{\mathrm{n}, \ell}}{(2 \ell+1)} \sum_{\mathrm{m}=0}^{\ell}\left(2-\delta_{\mathrm{m}, \mathrm{o}}\right) \delta_{\mathrm{m}, \mathrm{m}^{\prime}}\left|\left\langle\mathrm{n}^{\prime} \ell^{\prime} \mathrm{m}^{\prime}\left|\mathrm{l}^{\mathrm{i} \overrightarrow{\mathrm{Q}} \cdot \overrightarrow{\mathrm{r}}}\right| \mathrm{n} \ell \mathrm{m}\right\rangle\right|^{2},
$$

As in Equation (7), $|\mathrm{n} \ell \mathrm{m}\rangle$ is a hydrogenic wave function. The limits of integration in Equations (14) and (15) are:

$$
\begin{aligned}
& \mathrm{Q}_{1}=\frac{1}{2 \mathrm{v}}\left(\frac{1}{\mathrm{n}^{2}}-\frac{1}{\mathrm{n}^{\prime 2}}\right), \\
& \mathrm{Q}_{2}, \mathrm{Q}_{2}^{\prime} \approx 2 \mathrm{Z}_{\mathrm{n}, \mathrm{l}}^{-1} \mathrm{~K}_{\mathrm{i}} \rightarrow \infty,
\end{aligned}
$$

and

$$
\mathrm{Q}_{1}^{\prime}=\mathrm{Q}_{1}+\overline{\mathrm{I}}_{\mathrm{T}} /\left(2 \mathrm{Z}_{\mathrm{n}, \ell}^{2} \mathrm{v}\right)
$$


where $\overline{\mathrm{I}}_{\mathrm{T}}$ is an effective excitation energy for the target atom. For excitation of light atoms such as $\mathrm{H}, Q_{1}^{\prime}$ is sensitive to the value of $\overline{\mathrm{I}}_{\mathrm{T}}$ (Levy, 1969), which may be taken as the average excitation potential determined in the Bethe-theory of stopping power (see Dalgarno (1962) and Fano (1963) for a review). For H, $\overline{\mathrm{I}}_{\mathrm{T}}=15.0 \mathrm{eV}$; for He, $\overline{\mathrm{I}}_{\mathrm{T}}=41.8 \mathrm{eV}$. For highly charged heavy ions, it is apparent that the first term in Equation (19) will dominate the second; therefore, the expression for $Q_{1}^{\prime}$ will not be sensitive to the value of $\overline{\mathrm{I}}_{\mathrm{T}}$. This was found to be especially true for the excitation of Fe-ions.

For highly charged ions, $\mathrm{Z}_{\mathrm{n}, \ell} \gg 1$, then $\mathrm{Q}_{1} \gg \overline{\mathrm{I}}_{\mathrm{T}} /\left(2 \mathrm{Z}_{\mathrm{n}, \ell}^{2} \mathrm{v}\right)$ and $\mathrm{Q}_{1}^{\prime} \approx \mathrm{Q}_{1}$, also if $\mathrm{v} \gg 1 \mathrm{Q}_{1}^{\prime} \approx \mathrm{Q}_{1} \approx 0$. In either case, the approximate equality of the limits of integration in Equations (14) and (15) allows the integrands to be combined to give

$$
\sigma^{\mathrm{E}}(\mathrm{v}) \equiv \sigma_{\mathrm{e}}^{\mathrm{E}}+\sigma_{\mathrm{e}}^{\mathrm{I}} \approx 8 \pi \mathrm{a}_{0}^{2}\left[\frac{\mathrm{Z}_{\mathrm{T}}}{\mathrm{Z}_{\mathrm{n}, \ell^{\mathrm{v}}}^{2}}\right]^{2} \int_{\mathrm{Q}_{1}}^{\infty} \frac{\mathrm{dQ}}{\mathrm{Q}^{3}}\left[1+\mathrm{Z}_{\mathrm{T}}^{-1}-2 \mathrm{~F}\left(\mathrm{Z}_{\mathrm{n}, \ell} \mathrm{Q}\right)\right] \cdot \&_{\mathrm{n} \ell, \mathrm{n}^{\prime} \ell^{\prime}}(\mathrm{Q}) .
$$

For hydrogen targets Equation (14) becomes (Bates, 1962)

$$
\sigma^{\mathrm{E}}(\mathrm{v}) \approx 16 \pi \mathrm{a}_{0}^{2} \frac{1}{\left(\mathrm{Z}_{\left.\mathrm{n}, \ell^{\mathrm{v}}\right)^{2}}^{2}\right.} \int_{\mathrm{Q}_{1}}^{\infty} \frac{\mathrm{dQ}}{\mathrm{Q}^{3}}\left[1-\mathrm{F}\left(\mathrm{Z}_{\mathrm{n}, \ell} \mathrm{Q}\right)\right] \&_{\mathrm{n} \ell, \mathrm{n}^{\prime} \ell^{\prime}}(\mathrm{Q}),
$$

which is roughly a factor of two larger than $\sigma_{\mathrm{e}}^{\mathrm{E}}(\mathrm{v})$ of Equation $(15)$. For $\mathrm{v} \approx 1$, and for $Z_{n, l} \gg 1, F\left(Z_{n, l} Q_{1}\right) \rightarrow 0$, i.e. , for highly charged ions, the minimum momentum transfer for ionization is so large that, at that momentum, the target form factor is small and does not contribute significantly to the integral in Equation (21). 
For Fe-ions in atomic hydrogen we have found Equation (21) to be quite adequate for calculating excitation cross sections; however, in the case of O-ions, we found that the approximations made in obtaining Equation (21) were not justified, and we calculated $\sigma_{\mathrm{e}}^{\mathrm{E}}$ and $\sigma_{\mathrm{i}}^{\mathrm{E}}$ separately. Here we should point out that excitation by protons is approximately given by $\sigma_{\mathrm{e}}^{\mathrm{E}}(\mathrm{v})$ alone. In the case of excitation of O-ions, the present results are roughly a factor of two larger than those of Pravdo and Boldt (1975) which were calculated using only the formula for $\sigma_{\mathrm{e}}^{\mathrm{E}}(v)$, Equation (14).

Cascading from higher excited states to the upper level of the observed transition was also included in our calculation. For example, the total ( $1 \mathrm{~s} \rightarrow 2 \mathrm{p}$ ) excitation cross section was calculated as

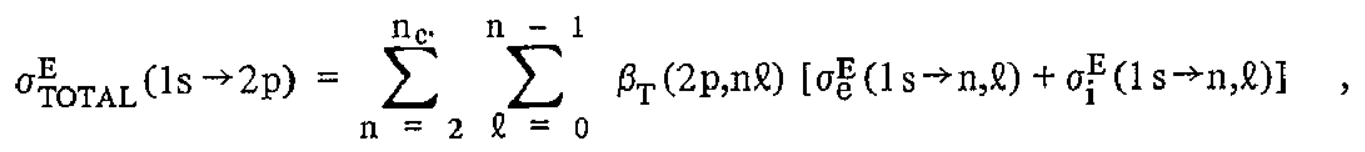

where $\beta_{\mathrm{T}}(2 \mathrm{p}, \mathrm{n} \ell)$ is the total branching ratio for $(\mathrm{n} \ell \rightarrow 2 \mathrm{p})$ transitions (Omidvar 1978), and where $\mathfrak{n}_{c} \equiv 6$, contributions for $\mathrm{n}>6$ being negligible.

Figure 3 contains examples of $\sigma_{\text {TOTAL }}^{\mathrm{E}}(1 \mathrm{~s} \rightarrow 2 \mathrm{p})$ calculated as described above. Cascading contributed $\sim 20 \%$ to the $\mathrm{O}^{+7}-\mathrm{H}$ and $\mathrm{O}^{+7}-\mathrm{He}$ cross sections at $0.1 \mathrm{MeV} /$ nucleon, while at $1.0 \mathrm{MeV} /$ nucleon cascading contributed $\sim 10 \%$ in each case. For $1 \mathrm{~s} \rightarrow 2 \mathrm{p}$ transition of $\mathrm{Fe}^{+25}$ in $\mathrm{H}$ (not shown), cascading contributed $\sim 20 \%$ to $\sigma_{\text {TOTAL }}^{\mathrm{E}}(1 \mathrm{~s} \rightarrow 2 \mathrm{p})$ at $1 \mathrm{MeV} /$ nucleon and $\sim 10 \%$ at $10 \mathrm{MeV} /$ nucleon, which is at the peak of the cross section. 
c) Charge-Exchange Cross Sections

In the passage of charged particles through gases, the main process for neutralizing the charged particles is the capture of electrons from the surrounding gas. If the surrounding gas is partially ionized, two other processes, dielectronic recombination and radiative recombination, can also contribute to the neutralization of the beam. The dielectronic recombination which is the inverse of the Auger effect can be neglected if the beam is made up basically of bare nuclei. Radiative recombination which is equivalent to the free-bound spontaneous emission has a cross section which is approximately a factor of ${ }^{\prime} \alpha^{3}$ smaller than the charge-exchange cross section, where $\alpha$ is the fine structure constant.

A fourth process which can occur with neutral surrounding gas is the radiative charge-exchange process (Briggs, et al. 1977; Butler, et al. 1977) but this is unimportant at energies of our interest.

There are numerous calculational methods applied to charge-exchange collisions, but they are mostly limited to charge exchange between protons and the simplest atoms, such as atomic hydrogen or helium. For highly charged ions charge-exchanging with multielectron atoms in the $\mathrm{MeV}$ energy range, where other than the $\mathrm{K}$-shell electrons contribute substantially to the chargeexchange cross sections, calculational methods are scarce. Aside from the Oppenheimer-Brinkman-Kramers approximation that will be discussed below, 
two other approximations can readily be applied to the multielectron case. One is the method of Jackson and Schiff for electron capture by protons from atomic hydrogen, where the interaction between the proton and the $\mathrm{F}$ nucleus is taken into account (Jackson and Schiff 1953). This method, with some modifications when applied to the $\mathrm{K}$-electron capture from He and $\mathrm{Ar}$, give satisfactory agreement with measurements (Omidvar, et al. 1976).

The other method that is being considered for application is the Bates' Atomic Expansion Method (Bates 1958). An important feature of this method is that it accounts for the lack of orthogonality of the initial and final atomic states. This method has been applied by Lin, et al. (1977) to the argon Kelectron capture by protons. Good agreement with measurement for both the total and the differential cross section is obtained.

The calculational method used here is the Oppenheimer-Brinkman-Kramers approximation (Mapleton 1972) modified by an empirical factor (Nikolaev 1967).

Let $\sigma\left(\mathrm{n}_{2} \ell_{2}, \mathrm{n}_{1} \ell_{1}\right)$ represent the charge exchange cross section for capture of an electron that is initially in a state of principal and azimuthal quantum number $n_{2} \ell_{2}$ of the target atom, and finally in a state $n_{1} \ell_{1}$ of the projectile. The cross section for population of the state $n_{0} l_{0}$ of the projectile is then given by

$$
\sigma_{\mathrm{T}}\left(\mathrm{n}_{2} \ell_{2}, \mathrm{n}_{0} \ell_{0}\right)=\sum_{\mathrm{n}_{1} \geqslant \mathrm{n}_{0}, \ell_{1}} \sigma\left(\mathrm{n}_{2} \ell_{2} ; \mathrm{n}_{1} \ell_{1}\right) \beta_{\mathrm{T}}\left(\mathrm{n}_{1} \ell_{1}, \mathrm{n}_{0} \ell_{0}\right)
$$


where $\beta_{\mathrm{T}}\left(\mathrm{n}_{1} \ell_{1}, \mathrm{n}_{0} \ell_{0}\right)$ are the electric dipole branching ratios for $\mathrm{n}_{1} \ell_{1} \rightarrow \mathrm{n}_{0} \ell_{0}$ transitions. The $\sigma\left(\mathrm{n}_{2} \ell_{2}, \mathrm{n}_{1} \ell_{1}\right)$ were calculated using the analytic form of the charge exchange amplitude for the hydrogenic wave functions given elsewhere (Omidvar 1975). Extensive tabulation of $\beta_{T}\left(n_{1} \ell_{1}, n_{0} \ell_{0}\right)$ is given by Omidvar (1978).

In Table 1 some recently measured cross sections are compared with related calculated cross sections. As is seen, the calculated charge-exchange cross sections are in fair agreement with measurement for $\mathrm{H}$ and $\mathrm{He}$ targets, but for atoms that have other than $\mathrm{K}$-shell electrons, the calculated cross sections are too large by a factor of 2-6, and in some exceptional cases this factor is as large as 23. In Section IV we will make an estimate of the change in our results if we reduce the charge exchange cross section by a factor of 5 .

The charge exchange cross sections are presented in Figures 4 and 5.

In a number of ways the present charge-exchange calculation is an improvement over the previous calculations (Serlemitsos, et al. 1973; Pravdo, et al. 1975; and Watson 1975). The most important improvement is to account for the contribution to the charge exchange cross section by the target atomic shells other than the K-shell, while these authors have consistently negelected the non-K-sheli electrons. From Figure 4 it is seen that the non-K-shell contribution to the cross section is substantial and in some cases dominate. An account of other improvements can be found in Bussard, et al. (1978). 


\section{CHARGE EQUILIBRIUM AND.RADIATION}

\section{a) Charge Fractions}

The ionization and charge exchange cross sections given in the last section were used to calculate the fraction of the ion-beam in each charge state as a function of energy. The rate of change of the fraction $\mathbf{F}_{Z}$ of a beam with charge $Z$ is given by (e.g., see Nikolaev 1965)

$$
\frac{d F_{z}}{d u}=\sum_{j \neq z} F_{j} \sigma_{j z}-F_{z} \sum_{j \neq z} \sigma_{Z j},
$$

where $\mathrm{du} \equiv \rho \mathrm{dx}$, with units of atoms $/ \mathrm{cm}^{2}$. Here $\mathrm{dx}$ is an interval of distance over which the velocity of the projectile can be considered constant and $\rho$ is the density of target atoms in atoms $/ \mathrm{cm}^{3}$. The cross section $\sigma_{\mathrm{jz}}$ is the probability of an ion initially of charge $j$ having a final charge $Z$ after interacting with a target atom. As $\mathfrak{u} \rightarrow \infty, d \mathfrak{d} / \mathfrak{d u}=0$, and an equilibrium charge distribution is established in the beam at each velocity. Under these conditions we can express the condition of equilibrium as

$$
\sum_{\substack{j \leqslant z \\ K} \mathrm{Z}} F_{j} \sigma_{j K}=\sum_{\substack{j>z \\ K \leqslant z}} F_{j} \sigma_{j K},
$$

If we assume an equilibrium charge distribution exists for low energy cosmic rays, then we have an equation like Equation (25) for each charge state Z. By further assuming that charge changing processes involving only one electron are dominant, Equation (25) reduces to

$$
\mathrm{F}_{\mathrm{Z}} \sigma_{\mathrm{Z}}^{\mathrm{I}}=\mathrm{F}_{\mathrm{Z}+1} \sigma_{\mathrm{Z}+1}^{\mathrm{C}}
$$


with $\sum_{Z} \mathrm{~F}_{\mathrm{Z}} \equiv 1$ and where

$$
\sigma_{\mathrm{Z}}^{\mathrm{I}} \equiv \sigma_{\mathrm{Z}, \mathrm{Z}+1} \text { and } \sigma_{\mathrm{Z}+1}^{\mathrm{C}} \equiv \sigma_{\mathrm{Z}+1, \mathrm{Z}}
$$

are the single electron ionization and charge exchange cross sections.

In Figure 6 we show the result of determining the charge fractions from Equation (26) for a beam of $\mathrm{O}$-ions in atomic hydrogen for charge states $\mathrm{O}^{+4}$ to $\mathrm{O}^{+8}$. Also shown is a similar result obtained for a mixture of gases representing the interstellar medium. For this case, effective ionization cross sections per $\mathrm{H}$ atom were calculated as

$$
\sigma_{Z}^{\mathrm{I}}=\sigma_{Z}^{\mathrm{I}}(\mathrm{H})+0.085 \sigma_{Z}^{\mathrm{I}}(\mathrm{He})
$$

where the helium cross section is multiplied by its abundance in the in terstellar medium relative to hydrogen. The effective charge exchange cross sections per $\mathrm{H}$ atom were taken to be

$$
\sigma_{Z}^{C}=\sum_{\mathrm{A}} \frac{\mathrm{N}_{\mathrm{A}}}{\mathrm{N}_{\mathrm{H}}} \sigma_{\mathrm{Z}}^{\mathrm{C}}(\mathrm{A}),
$$

i. e., the sum over the values for the most abundant elements times their abundances relative to hydrogen. The elements contained in the sum are those indicated in Figure 5. Since $\sigma^{I}$ scales roughly as the square of the target atom's nuclear charge, $\mathrm{Z}_{\mathrm{T}}^{2}$, while $\sigma^{\mathrm{C}}$ scales roughly as $\mathrm{Z}_{\mathrm{T}}^{3}$ to $\mathrm{Z}_{\mathrm{T}}^{5}$, less abundant elements contribute significantly to $\sigma^{\mathrm{C}}$, as discussed in Section II, while only He contributes significantly to $\sigma^{\mathrm{I}}$, Equation (28). Also shown for comparison are the set of charge fractions determined by Pravdo and Boldt (1975). The effect 
of their use of charge exchange cross sections which are too large by a factor of about 3 is to slightly shift the charge fractions toward higher energy.

In- Figure-7-the- set of charge fractions for a beam-of -Fe-ions-in-H atoms with charge states from $\mathrm{Fe}^{+16}$ to $\mathrm{Fe}^{+26}$ are given. Also shown are the charge fractions for the case of the interstellar mixture of gases. In this case $\sigma_{Z}^{I}$ was determined by scaling the elastic-and inelastic-target cross sections [Equations (2)-(4)] separately as:

$$
\sigma_{Z}^{I}=\sum_{N_{A}} \frac{N_{A}}{N_{H}}\left[Z_{A}^{2} \sigma_{\mathrm{e}}^{I}(H)+Z_{A} \sigma_{i}^{I}(H)\right],
$$

while the charge exchange cross sections were obtained as in Equations (23) and (29). The effect of the interstellar medium was to shift the charge fractions to higher energies by $\sim 3 \mathrm{MeV} / \mathrm{amu}$, and also to broaden the charge fraction curves. This broadening seems to be caused by the steeper slope of $\sigma_{Z}^{\mathrm{C}}$ in pure $H$ atoms as compared to $\sigma_{\mathrm{Z}}^{\mathrm{C}}$ in the interstellar mixture (see Fig. 5).

\section{b) Effective X-Ray Production Cross Sections}

The effective $\mathrm{X}$-ray production cross sections $\sigma_{\mathrm{X}}$ were calculated by multiplying the appropriate cross sections for excitation, ionization and charge exchange by the fractions $F_{Z}$ of the beam having a given charge state. For example, to calculate $\sigma_{\mathrm{x}}(0.65)$, the cross section for oxygen ions to produce $0.65 \mathrm{KeV} \mathrm{X}$-rays, we take

$$
\sigma_{\mathrm{X}}(0.65)=\mathrm{F}_{8} \sigma_{8}^{\mathrm{C}}(2 \mathrm{p})+\mathrm{F}_{7} \sigma_{7}^{\mathrm{E}}(1 \mathrm{~s} \rightarrow 2 \mathrm{p})
$$


where $\sigma_{8}^{\mathrm{C}}(2 \mathrm{p})$ is for capture of an electron by $\mathrm{O}^{+8}$ into the $2 \mathrm{p}$ state, including cascading from capture into higher levels.

In Figure 8 we have plotted $\sigma_{\mathrm{X}}(0.65), \mathrm{F}_{8} \sigma_{8}^{\mathrm{C}}(2 \mathrm{p})$, and $\mathrm{F}_{7} \sigma_{7}^{\mathrm{E}}$ (1, $\left.\rightarrow 2 \mathrm{p}\right)$ for $\mathrm{X}$-ray production by $\mathrm{O}$-ions in the interstellar medium. There it is seen that the contributions from charge exchange and excitation are nearly equal. This result seems to be in accord with that of Watson (1975), while it disagrees with the Pravdo and Boldt (1975) result that charge exchange dominates excitation by roughly an order of magnitude. The source of this discrepancy is probably their underestimation of $\sigma_{7}^{\mathbb{E}}(1 \mathrm{~s}-2 \mathrm{p})$ by a factor of two, as described in Section $\Pi$, and the overestimation of $\sigma_{8}^{\mathrm{C}}(2 \mathrm{p})$ caused by using unscaled Brinkman-Kramers cross sections, which are a factor of about 4 too large.

Figure 9 is a plot of the cross section for production of $\sim 0.57 \mathrm{KeV} \mathrm{X}$-rays by $\mathrm{O}$-ions in the interstellar medium. In this case we have taken

$$
\sigma_{\mathrm{X}}(\sim 0.57)=\mathrm{F}_{7} \sigma_{7}^{\mathrm{C}}(2 \mathrm{p})+\mathrm{F}_{6} \sigma_{6}^{\mathrm{E}}(1 \mathrm{~s}-2 \mathrm{p})
$$

Here we have neglected the contribution to $\sigma_{\mathrm{X}}(\sim 0.57)$ from inner shell ionization since the $\mathrm{K}$-shell fluorescence yield $\omega_{\mathrm{K}}$ is small. For example, for neutral oxygen $\omega_{\mathrm{K}} \approx 0.009$ (Bambynek, et al. 1972). We point out that the expression for $\sigma_{\mathrm{x}}$ in Equation (32) differs from that of Pravdo and Boldt (1975) by the inclusion of the last term, while it is identical to that of Watson (1975).

In Figure 10 we give the results for $\mathrm{X}$-ray production by $\mathrm{O}$-ions in atomic hydrogen for comparison with those of Figures 8 and 9 obtained with the 
interstellar medium. In Figure 10 both $\sigma_{\mathrm{x}}(0.65)$ and $\sigma_{\mathrm{x}}(\sim 0.57)$ are plotted. The contributions to $\sigma_{\mathrm{x}}(\sim 0.57)$ are as described by Equation (32).

Eigure 11 is a.plot of $\sigma_{x-}(6.97)$, the thefective $X$-ray_production cross section. for $\mathrm{Fe}$-ions in atomic hydrogen (labeled $\mathrm{H}$ ) and in the interstellar medium (labeled I). In both cases $\sigma_{\mathrm{x}}$ is given by

$$
\sigma_{\mathrm{X}}(6.97)=\mathrm{F}_{26} \sigma_{26}^{\mathrm{C}}(2 \mathrm{p})+\mathrm{F}_{25} \sigma_{25}^{\mathrm{E}}(1 \mathrm{~s} \rightarrow 2 \mathrm{p})
$$

where the notation is the same as in Equation (31). Although not shown, the contributions of the two terms in Equation (33) are roughly equal, as in the case of oxygen shown in Figure 8.

Figure 12 is a plot of $\sigma_{\mathrm{X}}(\sim 6.68)$, for X-ray production by Fe-ions in the interstellar medium,

$$
\sigma_{\mathrm{X}}(\sim 6.68) \approx \mathrm{F}_{25} \sigma_{25}^{\mathrm{C}}(2 \mathrm{p})^{-}+\mathrm{F}_{24} \sigma_{24}^{\mathrm{E}}(1 \mathrm{~s} \rightarrow 2 \mathrm{p})+\omega_{\mathrm{K}} \sigma_{24}^{\mathrm{I}}\left(1 \mathrm{~s}^{2}\right) \sum_{\mathrm{z}>22} \mathrm{~F}_{\mathrm{z}}
$$

In Equation (34) we have included a term which gives an estimate of the effect of inner shell ionization on $\sigma_{\mathrm{X}}(\sim 6.68)$. This estimate could be improved by replacing $\sigma_{24}^{\mathrm{I}}\left(1 \mathrm{~s}^{2}\right)$ by the true inner shell ionization cross section for each charge state, and by replacing the fluorescence yield for neutral $\mathrm{Fe}, \omega_{\mathrm{K}}$, by the values corresponding to each charge state $\omega_{\mathrm{K}}^{Z}$. Thus the last term in Equation (35) should be

$$
\sum_{z>22} \omega_{\mathrm{K}}^{\mathrm{Z}} \mathrm{F}_{\mathrm{Z}} \sigma_{\mathrm{Z}}^{\mathrm{I}}\left(1 \mathrm{~s}^{2}\right)
$$


however the quantities $\omega_{\mathrm{K}}^{\mathrm{Z}}$ are, to our knowledge, not available in the literature. For this reason our estimate in Equation $(34)$ uses $\omega_{\mathrm{K}} \approx 0.437$ which is an average yield for $\mathrm{Fe}^{+16}-\mathrm{Fe}^{+22}$. A more complete discussion of inner shell ionization contributions is given in the Appendix.

Figure 13 gives $\sigma_{\mathrm{X}}(\sim 6.68)$ for $\mathrm{X}$-ray production by Fe-ions in atomic hydrogen for comparison with Figure 12.

In Figure $14 \mathrm{a}$ and $\mathrm{b}$ we have plotted the effective $\mathrm{X}$-ray production cross section for the $3 \mathrm{p} \rightarrow 2 \mathrm{~s}$ transitions of Fe at $\sim 1.13$ to $1.17 \mathrm{KeV}$ and also for the $3 \mathrm{~d} \rightarrow 2 \mathrm{p}$ and $3 \mathrm{~s} \rightarrow 2 \mathrm{p}$ transitions at $\sim 0.7$ to $1.1 \mathrm{KeV}$. Figure $14 \mathrm{a}$ was obtained for the interstellar medium and Figure 14b for atomic hydrogen. These cross sections were obtained from the expressions

$$
\begin{aligned}
\sigma_{\mathrm{x}}(3 \mathrm{p} \rightarrow 2 \mathrm{~s}) & \approx \mathrm{F}_{23} \sigma_{23}^{\mathrm{E}}(2 \mathrm{~s} \rightarrow 3 \mathrm{p})+\mathrm{F}_{24}\left[\sigma_{24}^{\mathrm{C}}(2 \mathrm{~s})+\sigma_{24}^{\mathrm{C}}(2 \mathrm{p})\right] \\
& +\mathrm{F}_{22} \sigma_{22}^{\mathrm{E}}(2 \mathrm{~s} \rightarrow 3 \mathrm{p})+\mathrm{F}_{23}\left[\sigma_{23}^{\mathrm{C}}(2 \mathrm{~s})+\sigma_{23}^{\mathrm{C}}(2 \mathrm{p})\right]
\end{aligned}
$$

and

$$
\sigma_{\mathrm{X}}(3 \mathrm{~d}, 3 \mathrm{~s} \rightarrow 2 \mathrm{p}) \approx \sum_{\mathrm{Z}=16}^{21} \mathrm{~F}_{\mathrm{Z}} \sigma_{\mathrm{Z}}^{\mathrm{E}}(2 \mathrm{p} \rightarrow 3 \mathrm{~s}, 3 \mathrm{~d})+\sum_{\mathrm{Z}=17}^{22} \mathrm{~F}_{\mathrm{Z}} \sigma_{\mathrm{Z}}^{\mathrm{C}}(2 \mathrm{p})
$$

As in the previous cases, the interstellar mixture has the effect of broadening the energy range in which $\sigma_{\mathrm{x}}$ is significant. Here we have neglected contributions from inner shell ionization of the $2 p$ level, which should be small.

We should point out that, in Equations (36) and (37), the cross sections for capture by $\mathrm{Fe}^{+21}-\mathrm{Fe}^{+17}$ were obtained by scaling with respect to $\mathrm{Z}_{\mathrm{n}, \ell}$ as, e.g., 


$$
\sigma_{22}^{C}(2 \mathrm{p}) \approx\left(\frac{\mathrm{Z}_{2,1}}{\mathrm{Z}_{2,0}}\right)^{\alpha} \sigma_{23}^{\mathrm{C}}(2 \mathrm{p})
$$

where the $\alpha^{\prime}$ s for each case were determined from the calculated cross sections for $\sigma_{24}^{C}(2 p)$ and $\sigma_{23}^{C}(2 p)$ for each energy: For this reason $\sigma_{\mathrm{x}}(3 \mathrm{p} \rightarrow 2 \mathrm{~s})$ and $\sigma_{\mathrm{x}}(3 \mathrm{~d}$, $3 s \rightarrow 2 p$ ) should not be as reliable as the other effective X-ray production cross sections.

c) Multiplicities

Table 2 is a summary of results for the calculation of the total number of $\mathrm{X}$-rays produced by a cosmic ray nucleus as it slows down in atomic hydrogen or in the interstellar medium. For a given X-ray production process, the multiplicity, $\mathrm{M}$, is calculated as

$$
M \equiv \int_{\mathrm{E}_{\mathrm{f}}}^{\mathrm{E}_{1}} \sigma_{\mathrm{X}}(\mathrm{E})\left[-\frac{\mathrm{dE}}{\mathrm{dX}}\right]^{-1} \mathrm{dE},
$$

where $E_{i}$ and $E_{f}$ are the initial and final ion energies in $\mathrm{MeV} / \mathrm{amu}$ chosen such that $\sigma_{\mathrm{X}}(\mathrm{E}) \approx 0$ outside the range $\mathrm{E}_{\mathrm{f}}<\mathrm{E}<\mathrm{E}_{\mathrm{i}}$. The effective $\mathrm{X}$-ray production cross sections $\sigma_{\mathrm{x}}$ in $\mathrm{cm}^{2} /(\mathrm{H}$-atom) have been described in detail above; and $-\mathrm{dE} / \mathrm{dX}$ is the stopping power of the medium in $\mathrm{cm}^{2}-(\mathrm{MeV} / \mathrm{amu}) /(\mathrm{H}-\mathrm{atom})$, which is taken from Northcliffe and Schilling (1970) and from Barkas and Berger (1964).

Table 2 also contains results of calculations by Watson (1975), Pravdo and Boldt (1975), and Bussard, et al. (1977). In comparing the present results with those of previous workers, it should be noted that the processes contributing to $\sigma_{\mathrm{x}}$ differ among the various results cited. For example, in comparing the 
present calculation to that of Watson for an atomic hydrogen medium, we find that, in each case, the latter multiplicities exceed our values. This is probably a result of Watson's larger charge exchange cross sections and contradicts the conclusion of Belkic and McCarroll that Watson's results may be seriously underestimating the $\mathrm{X}$-ray production rate by $\mathrm{Fe}^{+24}$ and $\mathrm{Fe}^{+25}$. In compaxing our $\mathrm{O}-$ ion multiplicities with those of Pravdo and Boldt (1975), we find reasonable agreement; however this is misleading since the contribution to $\sigma_{\mathrm{x}}$ from charge exchange and excitation is quite different. The Pravdo-Boldt $\sigma_{\mathrm{x}}$ was dominated by the charge exchange contribution, which exceeded excitation by an order of magnitude (see Section II), while we find the two contributions to be comparable, in agreement with Watson. Our results for Fe-ions in the interstellar medium are in reasonable agreement with those of Bussard, et al. (1977), with respect to both the multiplicities and the nature of the cross section $\sigma_{\mathrm{x}}$, whose largest component comes from excitation of the ion, rather than charge exchange. 
IV. DISCUSSION

In this section we will consider the effect which overestimating charge exchange cross sections has on our final results for ion beams in the interstellar medium. "In addition, we discuss the effect of multielectron charge changing processes on the calculation of the equilibrium charge distribution. Finally, the contribution to $\mathrm{X}$-ray production from inner-shell ionization of neutral Fe in the interstellar medium by low energy cosmic ray ions will be discussed.

For the case of Fe-ions in atomic hydrogen, we have been able to compare our results for both ionization and charge exchange with the data of Berkner, et al. (1977). Agreement between calculated and experimental values was found to be within $\sim 25 \%$ for hydrogen targets. Similar agreement was also found for oxygen ions in helium. However, comparison of charge exchange cross sections for Fe-ions in argon indicates that the Brinkman-Kramers approximation scaled with the Nikolaev factor does not correctly reproduce the empirical values. Thus our effective $\mathrm{X}$-ray production cross sections for Fe-ions in the interstellar medium are not as reliable as they are for Fe-ions in hydrogen, since, for the former case, capture from less abundant atomic Fe and $\mathrm{O}$ was found to dominate.

We have estimated the effect of using charge exchange cross sections which may be a factor of $\sim 5$ too large for Fe-ions in the interstellar medium. In Figure 15 we give the results of calculating $\sigma_{X}(\sim 6.68)(\mathrm{Eq} .34$ ) after dividing the 
charge exchange cross sections by 5 . Thus the equilibrium charge fractions and capture cross sections of Equation (34) will be altered from the values used to obtain Figure 12. The multiplicity for $\sigma_{\mathrm{x}}(\sim 6.68)$ from Figure 15 is 5.2 which compares with 9.8 from Figure 12. Similar effects should occur for the câse of oxygen ions in the interstellar mixture.

Aside from possible errors in the values of the single electron ionization and charge exchange cross sections which were used to calculate the equilibrium charge fractions and $\mathrm{X}$-ray production cross sections, the processes involving multiple capture and loss of electrons may be important for the case of heavy targets. The data of Macdonald and Martin (1965) show that for $\mathrm{O}^{+8}$ in argon at 2. $5 \mathrm{MeV} / \mathrm{amu}$, double capture is about $15-20 \%$ of single capture. For $\mathrm{Fe}^{+20}$ $\mathrm{Fe}^{+26}$ in argon, in the range $1 \cdot 1-8.4 \mathrm{MeV} / \mathrm{amu}$, double capture is $\sim 20-30 \%$ of single capture, according to the data of Berkner, et al. (1977). For $\mathrm{O}^{+3}-\mathrm{O}^{+6}$ in argon at 1.1-2.5 MeV/amu, double loss is $\sim 10 \sim 25 \%$ of single loss. For lighter targets these effects are much smaller.

If one includes both one- and twowelectron charge changing processes in determining the equilibrium charge distribution, then Equation (25) reduces to the set of equations

$$
\mathrm{F}_{\mathrm{Z}-1} \sigma_{\mathrm{Z}-1, \mathrm{Z}+1}+\mathrm{F}_{\mathrm{Z}}\left[\sigma_{\mathrm{Z}, \mathrm{Z}+1}+\sigma_{\mathrm{Z}, \mathrm{Z}+2}\right]=\mathrm{F}_{\mathrm{Z}+1}\left[\sigma_{\mathrm{Z}+1, \mathrm{Z}}+\sigma_{\mathrm{Z}+1, \mathrm{Z}-1}\right]+\mathrm{F}_{\mathrm{Z}+2} \sigma_{\mathrm{Z}+2, \mathrm{Z}}
$$

one for each charge state $Z$. These equations can be put into a form similar to 
Equation (26), except that $\sigma_{Z}^{\mathrm{I}}$ and $\sigma_{\mathrm{Z}+1}^{\mathrm{C}}$ each become functions of several capture and loss cross sections:

$$
\mathrm{F}_{\mathrm{Z}} \sigma^{\mathrm{I}}=\mathrm{F}_{\mathrm{Z}+\mathrm{I}} \sigma^{\mathrm{C}}, \quad \sum_{\mathrm{Z}} \mathrm{F}_{\mathrm{Z}} \equiv 1
$$

We have found that the equilibrium charge fractions based on single electron loss are relatively insensitive to changes in the values of $\sigma_{Z}^{I}$ and $\sigma_{\mathrm{Z}+1}^{\mathrm{C}}$ (Eq. 26), as can be seen from Figures 6 and 7. Thus the reduction of Equations (40) to the form (41) suggests that as long as two electron charge changing processes are of the order of the one electron ones, the effect on the charge distribution of the beam should be small. However, we point out that when the target nuclear charge is greater than that of the projectile, multiple electron processes may dominate the one-electron ones and the above argument will then break down. Calculations of cross sections for multiple electron processes have been considered, for example, by Bottcher (1977).

Our crude estimate of the contribution to $\mathrm{X}$-ray production by inner shell ionization of Fe-ions indicates that this process does not significantly increase the effective production cross section; however a consideration of inner-shell. processes for the Fe-ions leads one to examine inner shell ionization of the neutral $\mathrm{Fe}$ in the interstellar medium. Although the abundance of Fe is $\sim 10^{-5}$ relative to hydrogen, the flux of protons and heavier ions which can ionize the $\mathrm{K}$ and $\mathrm{L}$ shells of neutral $\mathrm{Fe}$ is much greater than the flux of Fe-ions. The doppler broadening of this line will vary with the mass of the projectile. For 
ionization of $\mathrm{Fe}$ by protons, the line should be quite narrow, as opposed to the result of heavy particles in head on collisions.

The K-shell vacancy production rate is proportional to the sum of ionization and capture cross sections (Halpern and Law 1973, Lapicki and Losonsky 1977):

$$
\sigma=\sigma^{\mathrm{I}}+\sigma^{\mathrm{C}}
$$

For protons, $\sigma^{\mathrm{I}}$ is of order $10^{-20} \mathrm{~cm}^{2}$ at its peak value, while $\sigma^{\mathrm{C}}$ is of order $10^{-26} \mathrm{~cm}^{2}$ (see the curve labeled Is in Fig. 1). Thus $\sigma \approx \sigma^{\mathrm{I}}$ for protons; however $\sigma^{\mathrm{C}}$ rapidly increases for larger projectile charges, eventually dominating $\sigma^{\mathrm{I}}$, e.g. , for $\mathrm{O}^{+8}$ in argon, $\sigma^{\mathrm{C}} \approx \sigma^{\mathrm{I}}$ (Halperm and Law 1973). The values of $\sigma^{\mathrm{I}}$ can be obtained for heavier ions by scaling the results given by Rule for ionization of $\mathrm{Fe}^{+25}$ by protons. The appropriate $\sigma^{\mathrm{C}} \mathrm{s}$ can be determined from empirically scaled Brinkman-Kramers cross sections as discussed in Section II. Thus all of the data necessary to calculate the inner-shell ionization cross sections for $\mathrm{Fe}$ atoms by low energy cosmic ray ions are readily available. The effective $\mathrm{X}$-ray production cross section is then

$$
\sigma_{\mathrm{X}}=\omega_{\mathrm{K}} \sigma=\omega_{\mathrm{K}}\left(\sigma^{\mathrm{I}}+\sigma^{\mathrm{C}}\right),
$$

where $\omega_{\mathrm{K}}$ is the $\mathrm{K}$-shell fluorescence yield. A similar expression holds for $\mathrm{X}$-rays resulting from L-shell vacancy production. 


\section{CONCLUSTON}

We have used improved cross sections for charge exchange, ionization, and excitation of ions by neutral atoms, to calculate the charge equilibrium and radiation of an atomic beam passing through both atomic hydrogen and the interstellar medium. The present calculation results in a shift with respect to energy in the distribution of the charge fractions of an oxygen beam passing through interstellar matter. The magnitudes of different charge fractions are similarly changed.

We have also calculated the number of X-rays emitted per cosmic ray nucleus. We find charge exchange and excitation contribute comparably to X-ray production, while contributions from inner shell ionization are small. Partial agreement was obtained with the results of some authors; however, in some cases discrepancies were found. For example, we find our results for X-ray production by $\mathrm{Fe}^{+24}$ and $\mathrm{Fe}^{+25}$ in hydrogen to be about $1 / 3$ the value obtained previously by Watson.

Further improvement in the charge equilibrium and radiation calculation necessitates a more accurate knowledge of charge-exchange cross sections, especially for cases involving capture of $\mathrm{E}$ - and M-shell electrons. 
We are indebted to E. C. Sullivan for assisting us in programming, and providing us with his expert advice on numerous computational problems. We also thank R. Bussard for several helpful discussions. We are grateful to B. Crasemann for a discussion of fluorescence yields which was very illuminating and to F. W. Martin for providing us with his charge transfer data for oxygen ions in helium. 


\section{REFERENCES}

Allen, C. W. 1973, Astrophysical Quantities (3rd ed., London: The Athlone Press).

Bambynek, W., Crasemann, B., Fink, R. W., Freund, H. -V., Mark, H., Swift, C. D., Price, R. E., and Rao, P. Venugopala 1972, Rev. Mod. Phys., 44, p. 716.

Barkas, W. H. and Berger, M. J. 1964, Tables of Energy Losses and Ranges of Heavy Charged Particles, NASA SP-3013.

Bates, D. R. 1958, Proc. Roy. Soc., A247, p. 294. 1962, Atomic and Molecular Processes (New York: Academic Press), Ch. 14.

Belkié, Dž. and MeCarroll, R. 1977, J. Phys. B, 10, p. 1933.

Berkner, K. H., Graham, W. G., Pyle, R. V., Schlachter, A. S., and Stearns, J. W. 1977, Physics Letters A, 62A, 407.

Bhalla, C. P. 1973, Physics Letters 45A, p. 19. ' 1975a, J. Phys. B, 8, p. 2792. 1975b, Phys. Rev. A, 12, p. 122.

Briggs, J. S, and Dettmann, K, 1974, Phys. Rev. Letts., 33, p. 1123.

Bottcher, C. 1977, J. Phys. B, 10, p. L445.

Bussard, R. W., Omidvar, K., and Ramaty, R. 1978, Ap. J., 218.

Butler, S. E., Guberman, S. L., and Dalgarno, A. 1977, Phys. Rev. A, 16, p. 500 . 
Chen, M. H. and Crasemann, B. 1975, Phys. Rev. A, 12, 959.

Dalgarno, A. 1962, Atomic and Molecular Processes (New York: Academic Press), Ch. 15:

Dmitriev, I. S., Zhileǐkin, Ya. M., and Nikolaev, V. S. 1965, Zh. Eksp.

Teor. Fiz., 49, p. 500 (English trans1. in Soviet Phys. -JETP, 22, p. $352(1966))$.

Fano, U. 1963, Annual Review of Nuclear Science, 13, p. 1.

Halpern, A. M. and Law, J. 1973, Phys. Rev. Lett., $\underline{31}$, p. 4.

Hansen, J. 1973, Phys. Rev. A, $\underline{8}$, p. 822.

Lapicki, G. and Losonsky, W. 1977, Phys. Rev. A, 15, p. 896.

Levy II, H. 1969, Phys. Rev., 185, p. 7.

Lin, C. D., Soong, S. C., and Tunnell, L. N. 1977, Phys. Rev. A (to be published).

MacdonaId, J. R. and Martin, F. W. 1971, Phys. Rev. A, 4, 1965.

Mapleton, R. A. 1972, Theory of Charge Exchange (New York: John Wiley and Sons, Inc.).

McGuire, E. J. 1969, Phys. Rev., 185, p. 1.

Nikolaev, V. S. 1965, Usp. Fiz. Nauk, 85, p. 679 (English transl. Soviet Phys.

Uspekhi, $\underline{8}$, p. 269 (1965)). 1967, Soviet Physics JETP, 24, p. 847.

Northcliffe, L. C. and Schilling, R. F. 1970, Nuclear Data Tables, A7, p. 233.

Omidvar, K., Kyle, H. L. , and Sullivan, E. C. 1972, Phys. Rev. A, 5, p. 1174. 
Omidvar, K., Golden, J. E., McGuire, J. H., and Weaver, L. 1976, Phys.

Rev. A, 13, p. 500 .

Omidvar, K. 1978, Atomic Data and Nuclear Data (to be published). 1975, Phys. Rev. A, 12 , p. 911.

Pravdo, S. H. and Boldt, E. A. 1975, Ap. J., 200, p. 727.

Rule, D. W. 1977, Phys. Rev. A, 16, p. 19.

Senashenko, V. S., Nikolaev, V. S., and Dmitriev, I. S. 1968, Zh. Eksp. Teor.

Fiz., 54, p. 1203 (English transl. Soviet Phys. - JETP, 27, p. 643 (1968)).

Serlemitsos, P. J., Boldt, E. A., Holt, S. S., Ramaty, R., and Brisken, A.

F. 1973, Ap. J. (Letters), $\underline{184}$, p. L1.

Watson, W. D. 1975, Ap. J., 206, p. 842. 
Table 1

Comparison of some experimental and calculated cross sections and their ratios for a number of charge-exchange reactions. $E$ and $Z_{1}$ are the energies and charge numbers of the projectile. $\sigma(\mathrm{Ex})$ and $\sigma(\mathrm{CAL})$ are the experimental and calculated cross sections in units of $\mathrm{cm}^{2}$. The first three cases of charge exchange are due to Berkner, et al. (1977), and the last case is due to Macdonald and Martin (1971). Random experimental uncertainties for the first three cases are approximately $\pm 5 \%$, and systematic uncertainties for these cases are $\pm 7 \%$. For the last case the experimental error is $\sim 10-15 \%$.

\begin{tabular}{|c|c|c|c|c|}
\hline \multicolumn{5}{|c|}{$\mathrm{Fe}^{+\mathrm{Z}_{1}}+\mathrm{H} \rightarrow \mathrm{Fe}^{+\left(\mathrm{Z}_{1}-1\right)}+\mathrm{H}^{+}$} \\
\hline $\mathrm{E}(\mathrm{MeV} / \mathrm{amu})$ & $\mathrm{Z}_{1}$ & $\sigma(\mathrm{Ex})$ & $\sigma(\mathrm{CAL})$ & $\sigma(\mathrm{CAL}) / \sigma(\mathrm{Ex})$ \\
\hline 3.4 & 25 & $3.9-20 *$ & $3.49-20$ & 0.89 \\
\hline 3.4 & 24 & $3.6-20$ & $2.71-20$ & 0.75 \\
\hline 3.4 & 23 & $3.0-20$ & $2.36-20$ & 0.79 \\
\hline 3.4 & 22 & $2.7-20$ & $1.98-20$ & 0.73 \\
\hline 3.4 & 21 & $2.3-20$ & $1.71-20$ & 0.74 \\
\hline 3.4 & 20 & $2.2-20$ & $1.47-20$ & 0.67 \\
\hline 1.1 & 21 & $5.0-18$ & $4.72-18$ & 0.94 \\
\hline 1.1 & 20 & $4.0-18$ & $4.13-18$ & 1.0 \\
\hline \multicolumn{5}{|c|}{$\mathrm{Fe}^{+Z_{1}}+\mathrm{Ar} \rightarrow \mathrm{Fe}^{+\left(Z_{1}-1\right)}+\mathrm{Ar}^{+}$} \\
\hline $\mathrm{E}(\mathrm{MeV} / \mathrm{amu})$ & $\mathrm{Z}_{1}$ & $\sigma(\mathrm{Ex})$ & $\sigma(\mathrm{CAL})$ & $\sigma(\mathrm{CAL}) / \sigma(\mathrm{Ex})$ \\
\hline 8.4 & 26 & $5.8-18$ & $1.62-17$ & 2.8 \\
\hline 8.4 & 25 & $5.6-18$ & $1.44-17$ & 2.6 \\
\hline 3.4 & 25 & $4.5-17$ & $2.57-16$ & 5.7 \\
\hline 3.4 & 24 & $4.1-17$ & $1.91-16$ & 4.7 \\
\hline 3.4 & 23 & $3.7-17$ & $1.67-16$ & 4.5 \\
\hline 1.1 & 21 & $1.46-16$ & $3.42-15$ & 23.0 \\
\hline 1.1 & 20 & $1.43-16$ & $2.94-15$ & 21.0 \\
\hline \multicolumn{5}{|c|}{$\mathrm{Ar}^{+\mathrm{Z}_{1}}+\mathrm{Ar} \rightarrow \mathrm{Ar}^{+\left(\mathrm{Z}_{1}-1\right)}+\mathrm{Ar}^{+}$} \\
\hline $\mathrm{E}(\mathrm{MeV} / \mathrm{amu})$ & $\mathrm{Z}_{1}$ & $\sigma(\mathrm{Ex})$ & $\sigma(\mathrm{CAL})$ & $\sigma(\mathrm{CAL}) / \sigma(\mathrm{Ex})$ \\
\hline 8.4 & 18 & $2.4-18$ & $5.78-18$ & 2.4 \\
\hline 8.4 & 17 & $2.4-18$ & $4.96-18$ & 2.1 \\
\hline 3.4 & 16 & $2.3-17$ & $5.35-17$ & 2.3 \\
\hline \multicolumn{5}{|c|}{$\mathrm{O}^{+\mathrm{Z}_{1}}+\mathrm{He} \rightarrow \mathrm{O}^{+\left(\mathrm{Z}_{1-1}\right)}+\mathrm{He}^{+}$} \\
\hline $\mathrm{E}(\mathrm{MeV} / \mathrm{amu})$ & $\mathrm{Z}_{1}$ & $\sigma(\mathrm{Ex})$ & $\sigma(\mathrm{CAL})$ & $\sigma \mathrm{CAL}) / \sigma(\mathrm{Ex})$ \\
\hline 2.5 & 8 & $9.07-20$ & $7.49-20$ & 0.826 \\
\hline 2.5 & 7 & $3.31-20$ & $3.44-20$ & 1.04 \\
\hline 2.5 & 6 & $1.33-20$ & $1.04-20$ & 0.782 \\
\hline $2: 5$ & 5 & $1.05-20$ & $5.05-21$ & 0.481 \\
\hline 1.1 & 8 & $2.25-18$ & $2.49-18$ & 1.11 \\
\hline 1.1 & 7 & $1.56-18$ & $1.75-18$ & 1.12 \\
\hline 1.1 & 6 & $7.38-19$ & $7.26-19$ & 0.984 \\
\hline 1.1 & 5 & $5.37-19$ & $4.80-19$ & 0.894 \\
\hline 1.1 & 4 & $3.20-19$ & $2.69-19$ & 0.840 \\
\hline
\end{tabular}

*The notation $a-b$ means a $\times 10^{-b}$. 
Table 2

Number of X-Rays per Cosmic Ray Nucleus

\begin{tabular}{|c|c|c|c|c|c|c|c|}
\hline \multirow{2}{*}{ Transition } & \multirow{2}{*}{$\begin{array}{c}\text { X-Ray } \\
\text { Energy (KeV) }\end{array}$} & \multicolumn{2}{|c|}{ Present Calc. } & \multirow{2}{*}{$\begin{array}{c}\text { Watson (1975) } \\
\mathrm{H}\end{array}$} & \multicolumn{2}{|c|}{ Pravdo-Boldt (1975) } & \multirow{2}{*}{$\begin{array}{c}\text { Bussard, et a1. (1977) } \\
\text { I }\end{array}$} \\
\hline & & $\mathrm{H}^{(\mathrm{a})}$ & $\mathrm{I}^{(\mathrm{b})}$ & & $\mathrm{H}$ & I & \\
\hline $\mathrm{O}^{+7}(2 \mathrm{p} \rightarrow 1 \mathrm{~s})$ & 0.65 & 21 & $49^{(d)}$ & & 21.91 & $42.75^{(\mathrm{d})}$ & \\
\hline$O^{+6}(2 p-1 s)$ & $\sim 0.57$ & 31 & $49^{(d)}$ & & 18.34 & $47.21(\mathrm{e})$ & \\
\hline $\mathrm{O}^{+6}-\mathrm{O}^{+7}$ & $\sim 0.57-0.65$ & 52 & 98 & $77(\mathrm{~d})$ & 40.20 & 89.96 & \\
\hline $\mathrm{Fe}^{+25}(2 \mathrm{p} \rightarrow 1 \mathrm{~s})$ & 6.97 & 1.4 & $6.5^{(\mathrm{d})}$ & & & & \\
\hline $\mathrm{Fe}^{+24}(2 \mathrm{p} \rightarrow 1 \mathrm{~s})$ & $\sim 6.5-6.7$ & 2.3 & $9.8^{(\mathrm{c})}$ & & & & \\
\hline $\mathrm{Fe}^{+24}-\mathrm{Fe}^{+25}$ & $\sim 6.5-6.97$ & 3.7 & 16.3 & $11(\mathrm{~d})$ & & & $10.6(\mathrm{~d})$ \\
\hline $\mathrm{Fe}^{+23}-\mathrm{Fe}^{+22}(3 \mathrm{p}-2 \mathrm{~s})$ & $\sim 1.1$ & 43 & $117^{(\mathrm{d})}$ & & & & \\
\hline $\mathrm{Fe}^{+21}-\mathrm{Fe}^{+16}(3 \mathrm{~s}, 3 \mathrm{~d} \rightarrow 2 \mathrm{p})$ & $\sim 0.7-1.0$ & 182 & 464 & & & & \\
\hline $\mathrm{Fe}^{+16}-\mathrm{Fe}^{+23}$ & $\sim 0.7-1.1$ & 225 & 581 & $310^{(d)}$ & & & \\
\hline
\end{tabular}

(a) Calculated for atomic hydrogen medium.

(b) Calculated for the interstellar medium.

(c) Includes contributions from charge exchange, excitation and ionization.

(d) Contains contributions from excitation and charge exchange.

(e) Includes charge exchange only. 


\section{APPENDIX}

Since $\mathrm{K}$-shell fluorescence yields $\omega_{\mathrm{k}}^{\mathrm{Z}}$ for the ions $\mathrm{Fe}^{\mathrm{tz}}$ are not available, we have fitted the results of theoretical calculations for the "ground state" configurations of the form $1 \mathrm{~s}(2 \mathrm{~s})^{2}(2 \mathrm{p})^{\mathrm{n}}$. For a given value of $n$, we have done a Ieast squares fit to the function

$$
\left\{\omega_{n}\left(Z_{p}\right) /\left[1-\omega_{n}\left(Z_{p}\right)\right]\right\}^{1 / 4}-C Z_{p}^{3}=A_{n}+B_{n} Z_{p}
$$

The constant $c$ was taken to be equal to the value obtained by fitting the data for fluorescence yields of neutral atoms with one $\mathrm{K}$-shell vacancy with the form (AI) (see Bambynek et al.), $\mathrm{c} \equiv-(0.64 \pm 0.07) \times 10^{-6}$. In $(A I) \omega_{n}\left(Z_{p}\right)$ is the $K-$ shell fluorescence yield for an ion or atom of nuclear charge $Z_{p}$ in the $1 s(2 s)^{2}(2 p)^{n}$ configuration. As input data, we have used $\omega_{\mathrm{k}}$ 's for neutral $\mathrm{B}, \mathrm{C}, \mathrm{N}$, and $\mathrm{O}$ (see Bambynek et a1. for references), as well as for various ions: $\mathrm{N}^{+\mathrm{Z}}(\mathrm{Z}=1-$ 2) (Bhalla 1975a), $\mathrm{Ne}^{+\mathrm{z}}(\mathrm{Z}=0 \rightarrow+5$ ) (Bhalla 1975b, Chen and Crasemann 1975), $\mathrm{Al}^{+\mathrm{Z}}(\mathrm{Z}=3-8)$ (McGuire 1969), and $\operatorname{Ar}^{+\mathrm{z}}(\mathrm{Z}=8-13)$ (Bhalla 1973). We note that $\mathrm{Z}$ indicates the ion's charge before creation of the $\mathrm{K}$-vacancy.

The term "ground state" used above means that we assume the projectile ion has enough time between collisions to go into the ground state, and further more we assume that creation of a $\mathrm{K}$-shell vacancy does not excite the outer electrons. For ionization by atomic hydrogen in the interstellar medium, these assumptions should be valid. If the target medium becomes denser, and heavier species of target atoms become more abundant, the above assumptions break 
down. In this case, a projectile with a given charge state may contain several configurations (Chen and Crasemann 1975). For example $\mathrm{Ne}^{+7}$ may contain the configurations $(1 s)^{1}(2 s)^{2} 2 p^{0},(1 s)^{1}(2 s)^{1}(2 p)^{1}$, and $(1 s)^{1}(2 s)^{0}(2 p)^{2}$. The fluorescence yields averaged over the multiplets of each configuration are $0,0.237$, and 0.238. After averaging these over the three configurations, assuming statistical weighting proportional to the number of multiplets for each configuration, a final value of $\omega_{\mathrm{k}}^{\mathrm{z}}=0.229$ is obtained (Chen and Crasemann 1975). Thus in this case the "ground state" result would be $\omega_{k}=0$, but the statistically averaged result is quite large.

Using the statistically averaged fluorescence yields for $\mathrm{Ne}$-ions (Chen and Crasemann 1975) and for $\mathrm{N}$-ions (Bhalla 1975a), we have again fit the function (AI) and determined $A_{n}$ and $B_{n}$. Since for each $n$ only two data points are available, this procedure is not very reliable.

Table 3 lists the values of the parameters $A_{n}$ and $B_{n}$ of eq. (A.1) for the charge states arising from the ground-state configurations $(1 s)^{1}(2 s)^{2}(2 p)^{n}$ and from statistically averaging over possible L-shell configurations. Also given are the estimated values of fluorescence yields for Fe-ions obtained by using the given $A_{n}$ and $B_{n}$ and letting $Z_{p} \rightarrow 26$ in (A1).

In Fig. 16 the $\omega_{n}(26)$ of Table 3 are plotted as a function of ion charge (before the $\mathrm{K}$-vacancy is produced).

To estimate the effect of the different assumptions concerning the state of the ion containing the $K$-shell vacancy, in Fig. 17 we have plotted the $X$-ray 
production cross sections for $\sim 6.5-6.8 \mathrm{KeV}$ X-rays by Fe-ions. In Fig. 17a, we give the results $\mathrm{Fe}-$ ions in atomic hydrogen assuming ions in the ground state just before and after $\mathrm{K}$-vacancy production (dashed line) and assuming the statistical distribution of $\mathrm{L}$-shell vacancies after production of the $\mathrm{K}$-shell vacancy (solid line). The multiplicities for these two cases are 2.3 and 2.5 , respectively. In Fig. 17b similar results are obtained for $\mathrm{Fe}$ in the interstellar medium. In this case the multiplicities are 9.8 (ground state configuration) and 11.3 (statistical distribution). In all cases the cross sections are calculated as in eq. (34) with expression (35) substituted for the last term.

From Fig. 17 it is seen that the inner-shell ionization contribution to X-ray production can be appreciable in the case of ions with a statistical distribution of L-shell vacancies. The correct fluorescence yields to use will depend on the assumptions made concerning the target density. 
Table 3

The coefficients $A_{n}$ and $B_{n}$ of eq. (A1) and the fluorescence yields obtained for Fe-ions when $Z_{p} \rightarrow 26$ in (A1). The subscript $n$ indicates the number of $2 p$ electrons in the "ground state" configuration $(1 S)^{1}(2 S)^{2}(2 p)^{n}$.

\begin{tabular}{ccccccc}
\hline & & \multicolumn{3}{c}{$\begin{array}{c}\text { Ground State } \\
\text { Configuration }\end{array}$} & & \multicolumn{3}{c}{$\begin{array}{c}\text { Statistically Averaged- } \\
\text { Configurations }\end{array}$} \\
\hline $\mathrm{n}$ & $\mathrm{A}_{\mathrm{n}}$ & $\mathrm{B}_{\mathrm{n}}$ & $\omega_{\mathrm{n}}(26)$ & $\mathrm{A}_{\mathrm{n}}$ & $\mathrm{B}_{\mathrm{n}}$ & $\omega_{\mathrm{n}}(26)$ \\
0 & 0 & 0 & 0 & $-1.34-1$ & $8.72-2$ & 0.953 \\
1 & $2.27-2$ & $3.61-2$ & 0.442 & $5.87-2$ & $4.96-2$ & 0.762 \\
2 & $3.41-2$ & $3.64-2$ & 0.468 & $2.42-2$ & $4.25-2$ & 0.610 \\
3 & $3.75-2$ & $3.56-2$ & 0.450 & $-3.0-3$ & $4.03-2$ & 0.532 \\
4 & $1.73-2$ & $3.61-2$ & 0.443 & & & \\
5 & $3.09-2$ & $3.50-2$ & 0.436 & & & \\
6 & $7.46-2$ & $3.17-2$ & 0.381 & & & \\
\hline
\end{tabular}




\section{FIGURE CAPTIONS}

Figure 1. Total ionization cross sections for Fe-ions of charge $i=+20-+25$ being ionized by $\mathrm{H}$-atoms. The solid curves are the present FBA results in $\mathrm{cm}^{2}$ as a function of energy in $\mathrm{MeV} /$ nucleon. The dashed curves are the BEA results for ionization by protons, and the data are from Berkner, et al. (1977).

Figure 2. Total ionization cross sections for 0 -ions of charge $\mathbf{i}=+3-+7$ being . ionized by H-atoms. The solid curves are from the FBA calculation and the dashed curves are the result of using the BEA for protons as targets.

Figure 3. Total excitation cross sections for the is $\rightarrow 2 p$ transition in $\mathrm{O}^{+7}$ colliding with $\mathrm{H}, \mathrm{H}^{+}$, and $\mathrm{He}$ and for $\mathrm{O}^{+6}$ colliding with $\mathrm{H}$. Cascading from higher excited levels is included as in Equation (22).

Figure 4. Electron capture by protons from different shells of neutral iron. The incident proton is in the energy range $5-40 \mathrm{MeV}$. It is seen that in the few MeV energy range, capturerfrom the $\mathrm{K}-$ shell has the lowest cross section. At high energies capture from the $\mathrm{K}$-shell dominates, as is expected theoretically.

Figure 5. Cross sections for electron capture by a beam of $\mathrm{Fe}^{+26}$ from $\mathrm{H}, \mathrm{He}$, $\mathrm{C}, \mathrm{N}, \mathrm{O}, \mathrm{Ne}, \mathrm{Mg}, \mathrm{Si}, \mathrm{S}$, and $\mathrm{Fe}$, and the total cross section. Cross 
section for each atom $A$ is multiplied by $N_{A} / N_{H}$, which is the relative interstellar abundance of atom A relative to atomic hydrogen. Values of $\mathrm{N}_{\mathrm{A}}$ and $\mathrm{N}_{\mathrm{H} .}$ are taken from Allen (1973).

Figure 6. Fractions of a beam of oxygen ions in hydrogen and in a mixture of gases representing the interstellar medium. The beam fractions in charge states $\mathrm{O}^{+8}$ to $\mathrm{O}^{+4}$ are plotted as a function of energy: present calculation, - - - Pravdo-Boldt (1975). The curves are labeled by the number of electrons on the ion in that charge state.

Figure 7. Fractions of a beam of Fe-ions in hydrogen and in the interstellar medium with charge states $\mathrm{Fe}^{+26}$ to $\mathrm{Fe}^{+16}$, as a function of energy. The curves are labeled by the number of electrons on the ion in that charge state.

Figure 8. Effective cross section for production of $0.65 \mathrm{KeV} \mathrm{X}$-rays by $\mathrm{O}^{+7}$. in the interstellar medium as a function of ion energy in $\mathrm{MeV} / \mathrm{amu}$.

Figure 9. Effective cross section for production of $\sim 0.57 \mathrm{KeV}$ X-rays by $\mathrm{O}^{+6}$-ions the interstellar medium.

Figure 10. Effective X-ray production cross sections for O-ions in hydrogen as a function of ion energy. 
Figure 11. Effective cross section for production of $6.97 \mathrm{KeV} \mathrm{X}$-rays by $\mathrm{Fe}^{+25}$ in hydrogen $(\mathrm{H})$ and in the interstellar medium $(\mathrm{I})$, as a function of ion energy in $\mathrm{MeV} / \mathrm{amu}$.

Figure 12. Effective cross section for production of $\sim 6.5-6.68 \mathrm{KeV}$ X-rays by Fe-ions in the interstellar medium.

Figure 13. Effective cross section for production of $\sim 6.5-6.68 \mathrm{KeV} \mathrm{X}$-rays by Fe-ions in hydrogen.

Figure 14. X-ray production cross sections for $\mathrm{Fe}^{+23}-\mathrm{Fe}^{+16}$ in (a) the interstellar medium and (b) hydrogen.

Figure 15. Effective cross section for production of $\sim 6.5-6.8 \mathrm{KeV} \mathrm{X}$-rays by Fe-ions in the interstellar medium. This case differs from Figure 12 in that all capture cross sections are reduced by a factor of 0.2 . The multiplicity becomes 5.2 , which compares with 9.8 from Figure 12 .

Figure 16. Estimated K-shell fluorescence yields for Fe-ions with charge states $+16-+22$. The upper curve results from assuming a statistical distribution of vacancy states in the L-shell, for a given charge state. The lower curve is obtained if the ion remains in the "ground" state after a $\mathrm{K}-$ shell vacancy is created. 
Figure 17. A comparison of the effects of using the two fluorescence yield curves of Figure 16 to determine the inner-shell ionization contribution to the production of $\sim 6.5-6.8 \mathrm{KeV} \mathrm{X}$-rays. Part a) is . the cross section for a hydrogen medium and part b) gives the results for the interstellar medium. The solid curves correspond to the use of fluorescence yields for a statistical distribution of vacancy states, while the dashed curves are for the yields from a purely "ground" state configuration. 


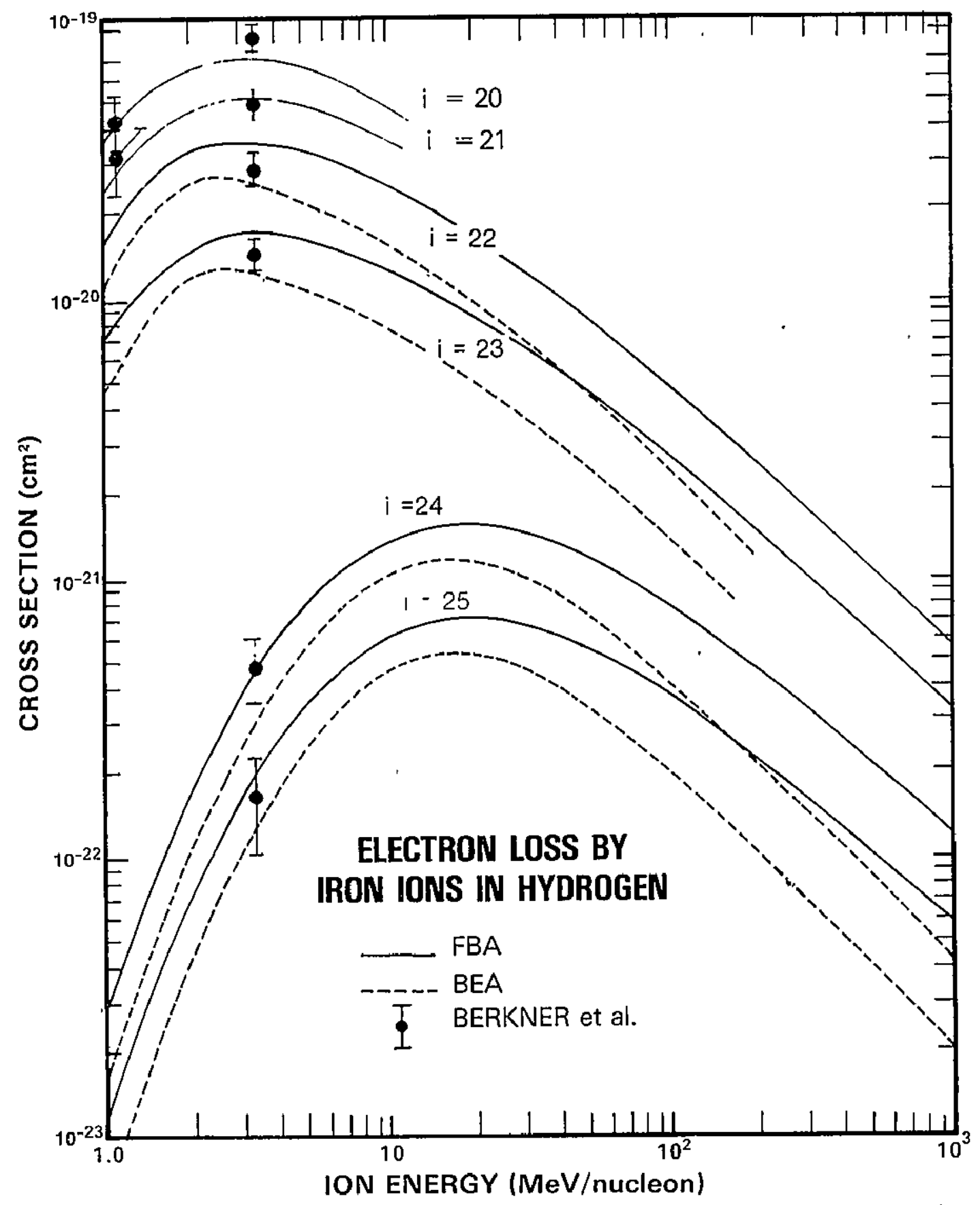

Figure 1 


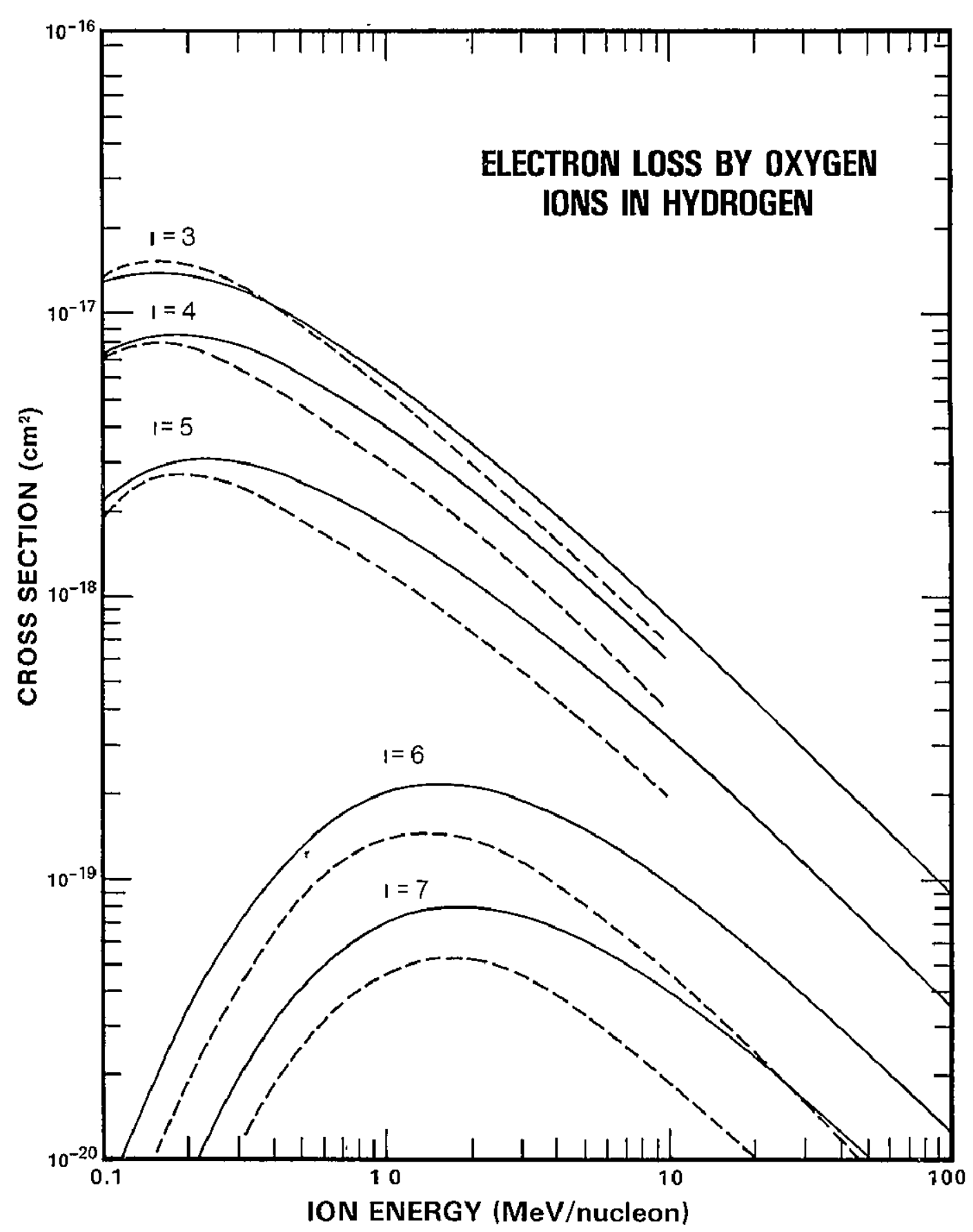

Figure 2 


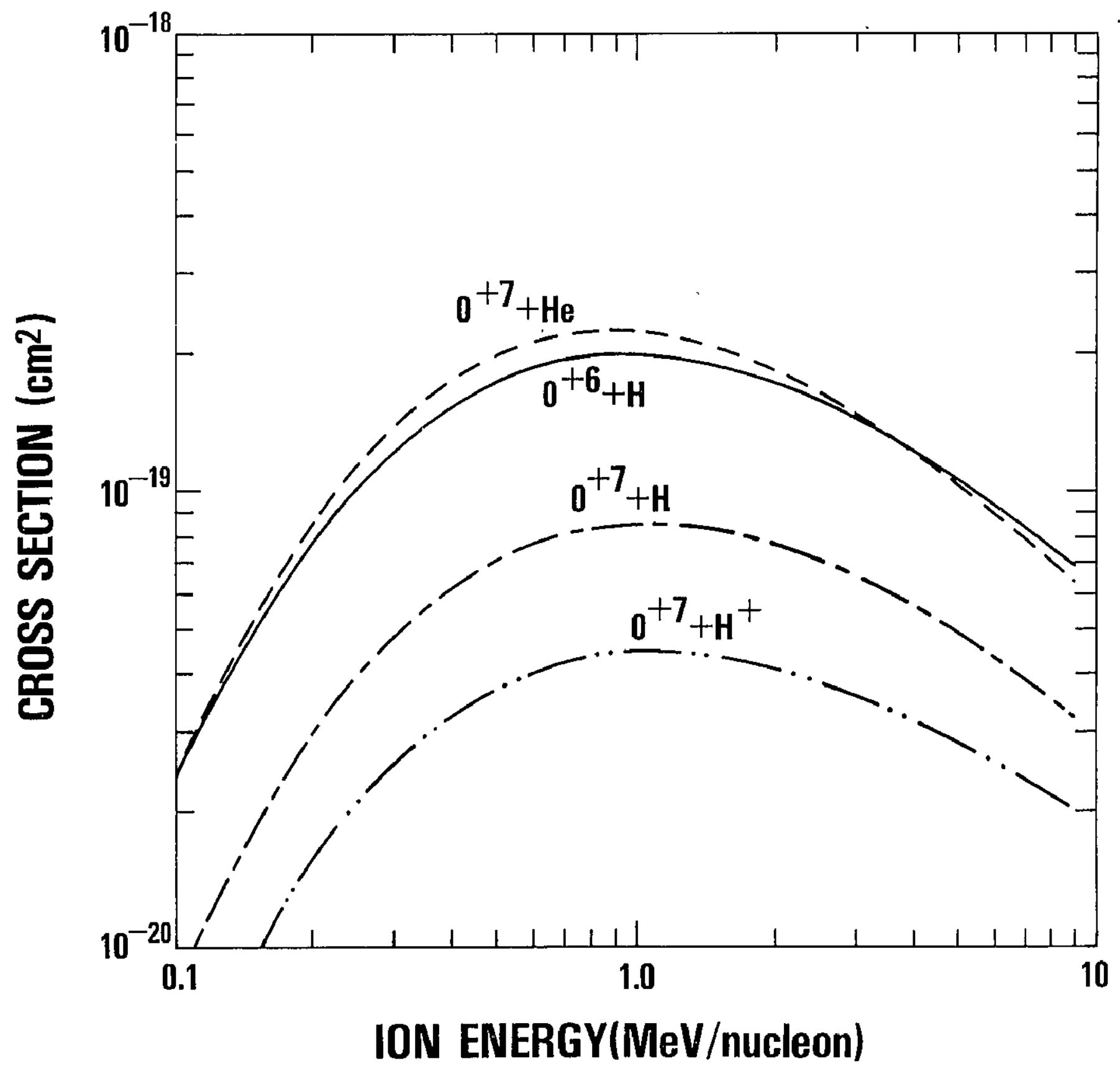

Figure 3 


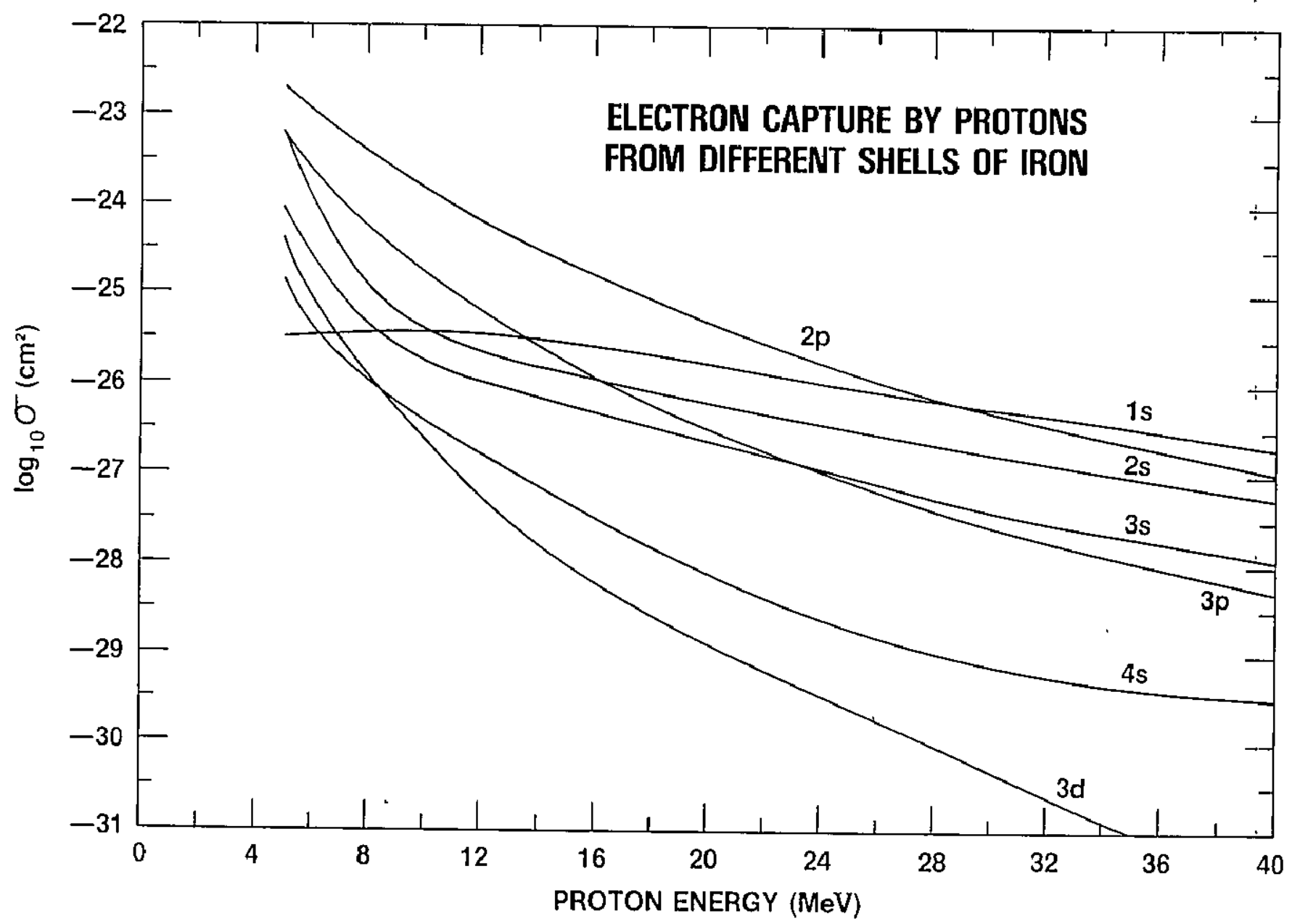

Figure 4 


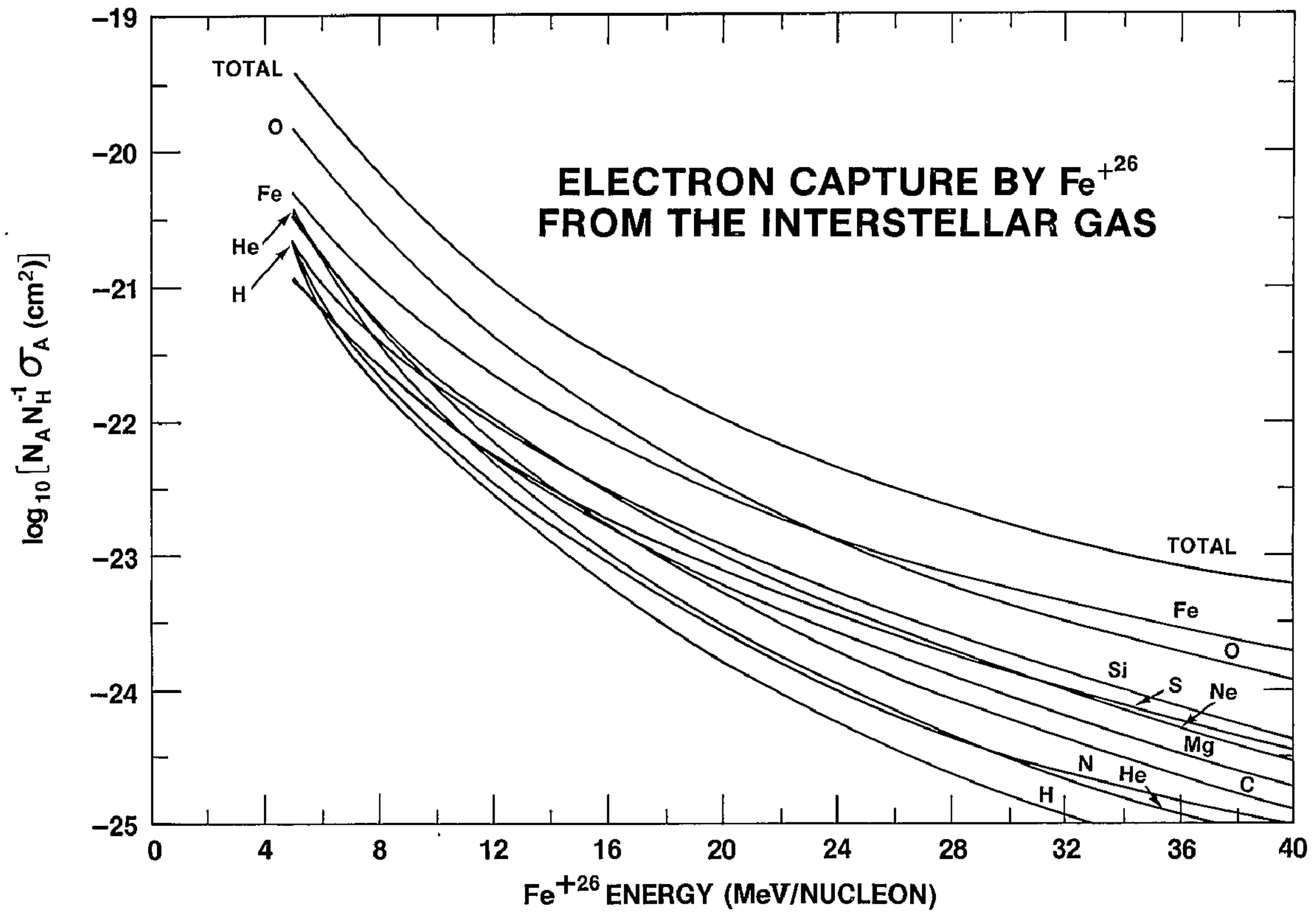

Figure 5 


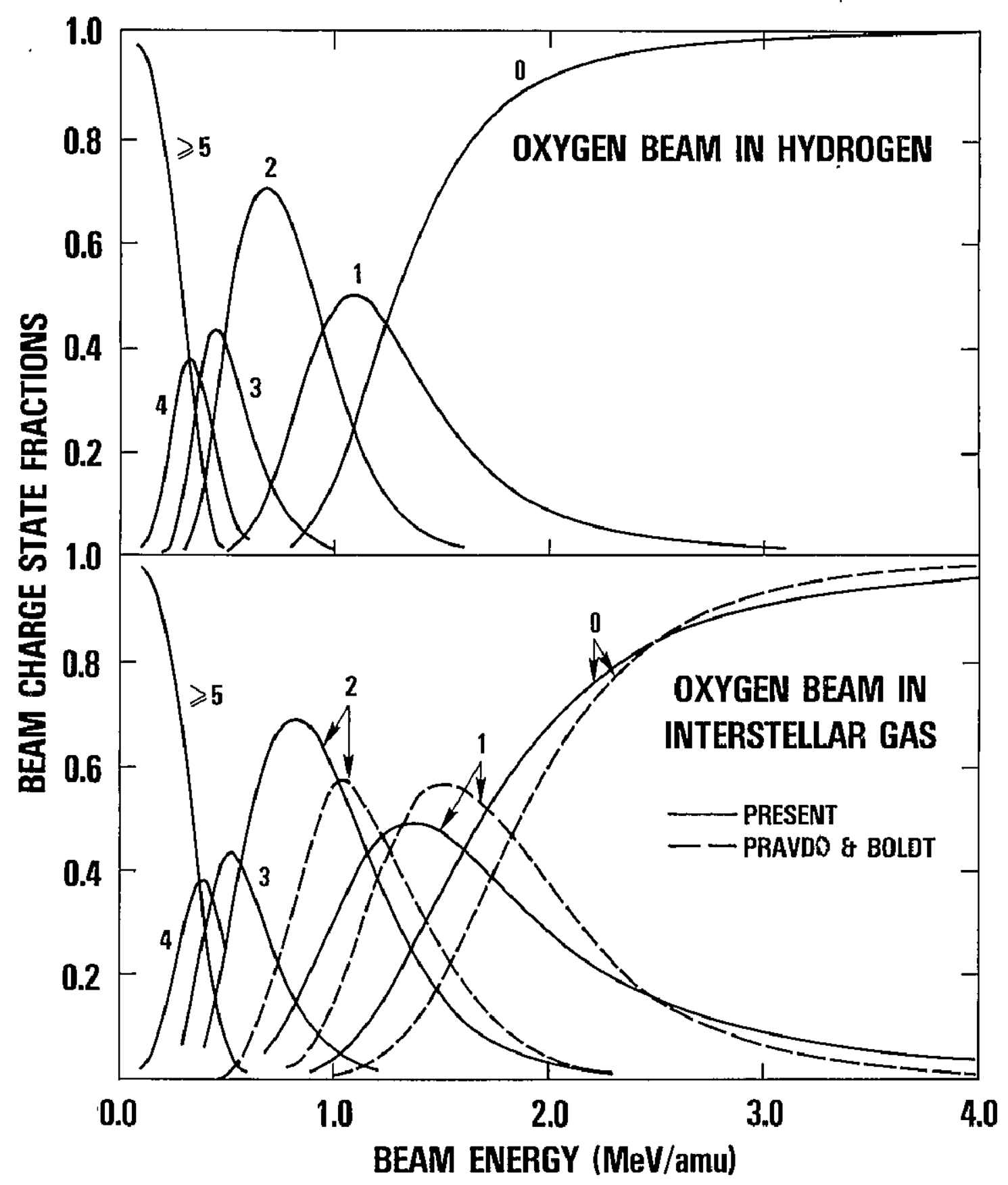

Figure 6 


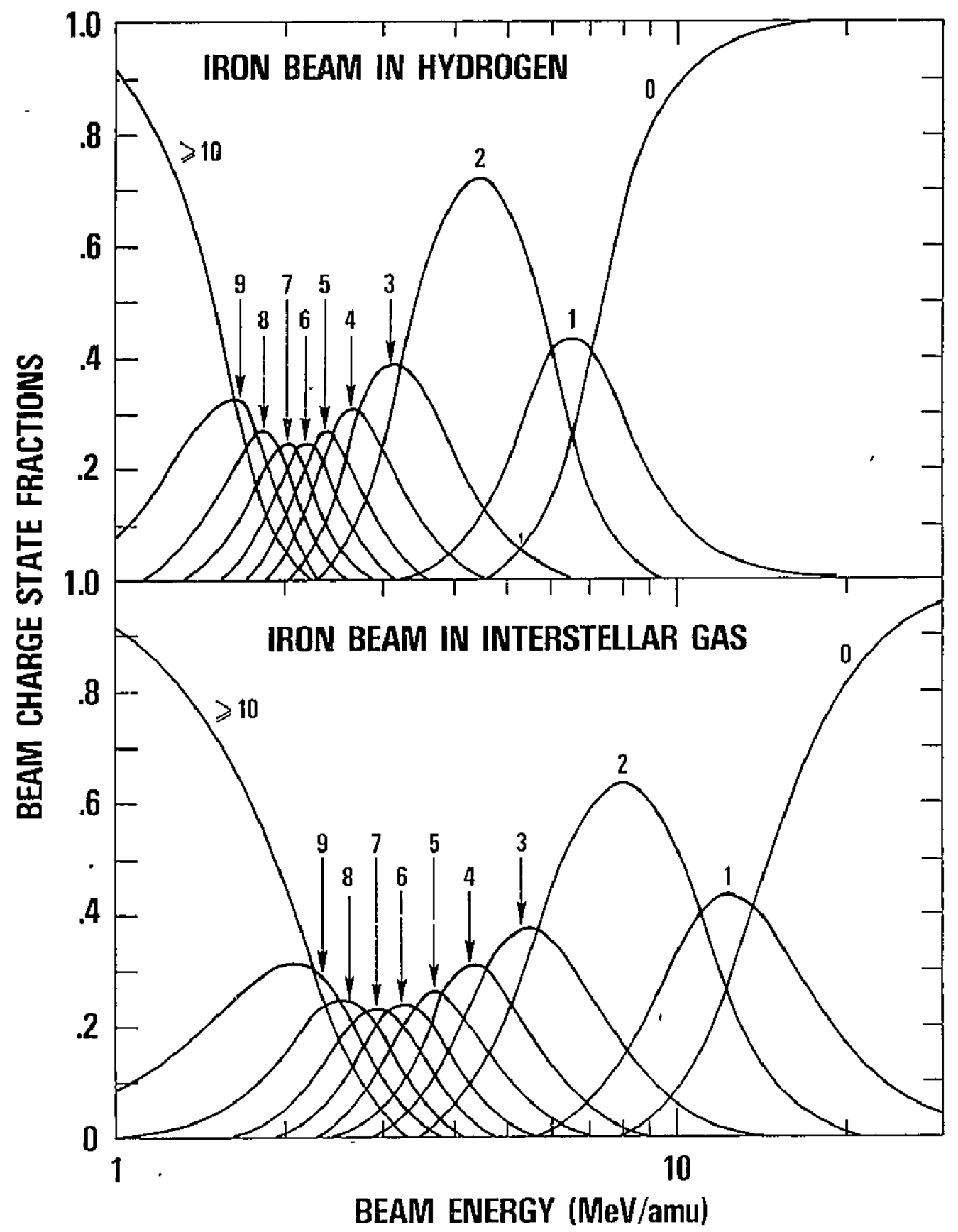

Figure 7 


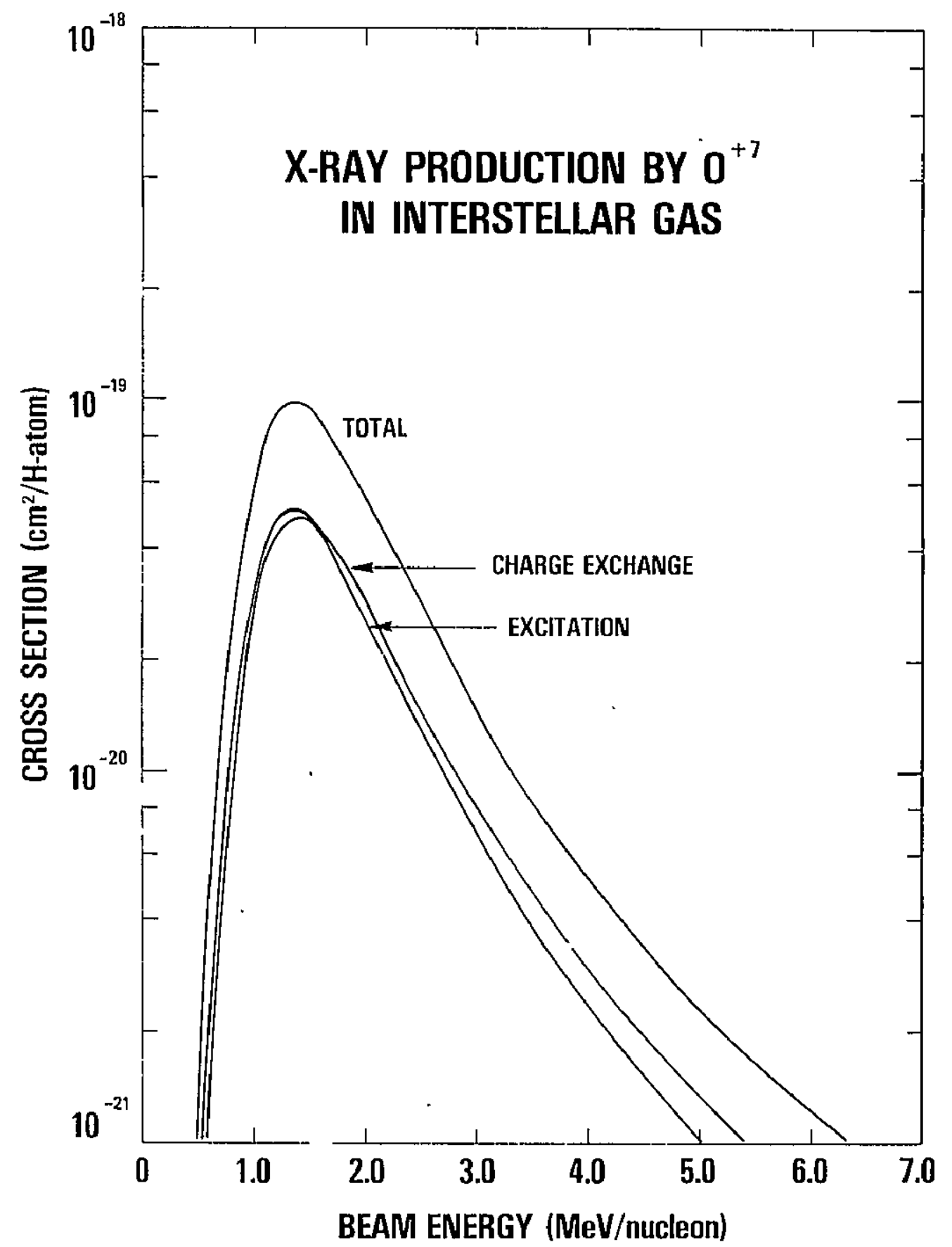

Figure 8 


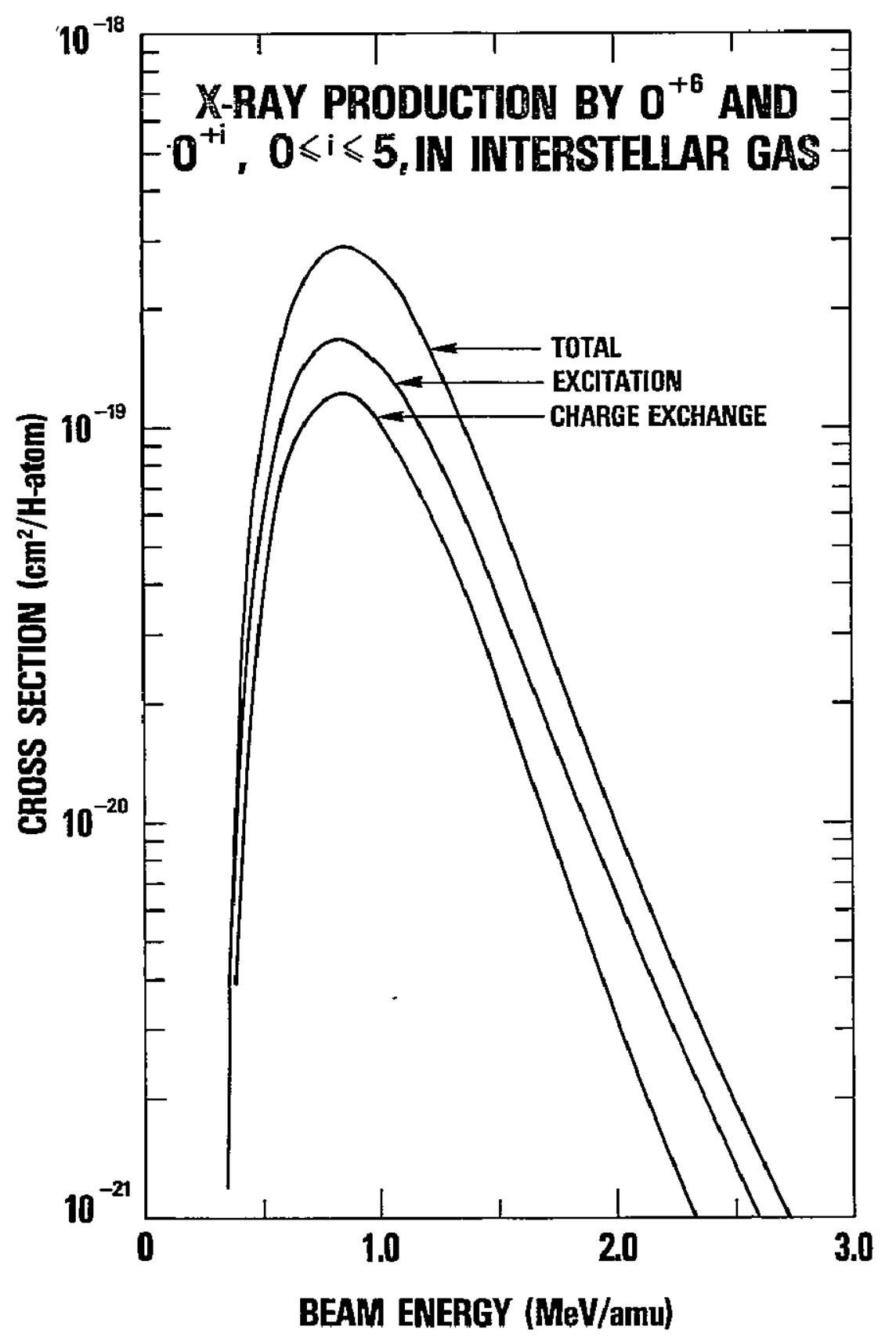

Figure 9 


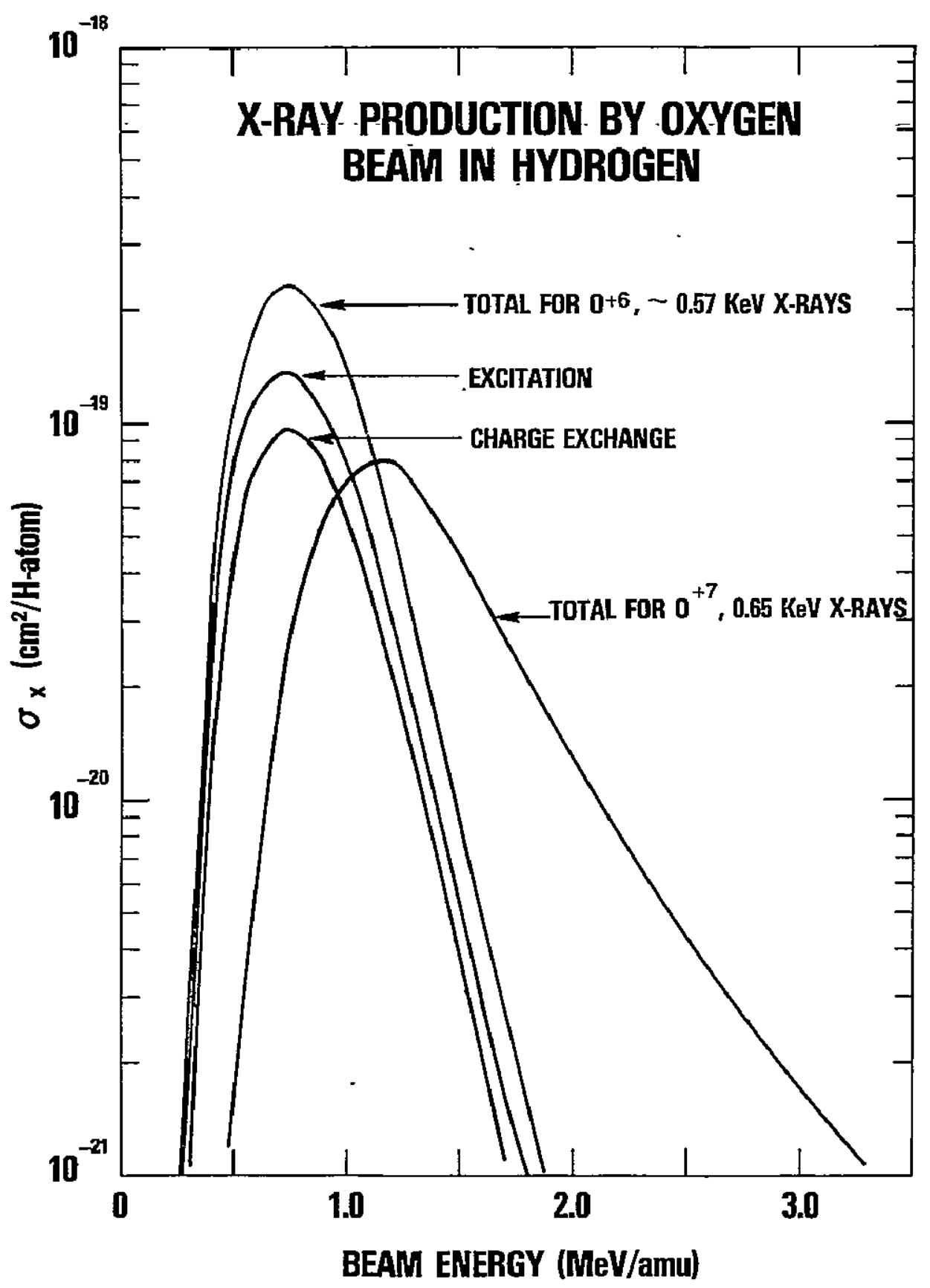

Figure 10 


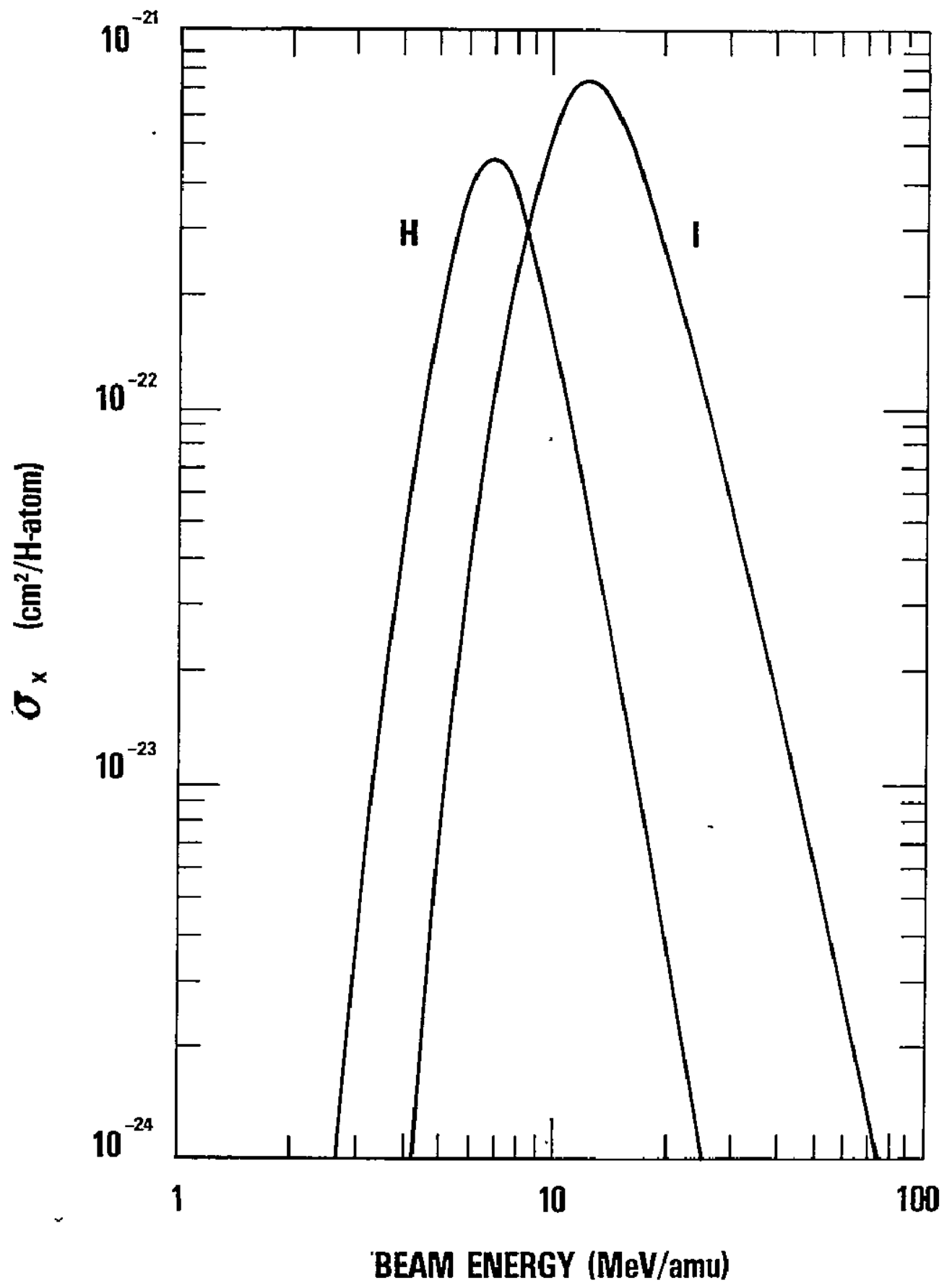

Figure 11 


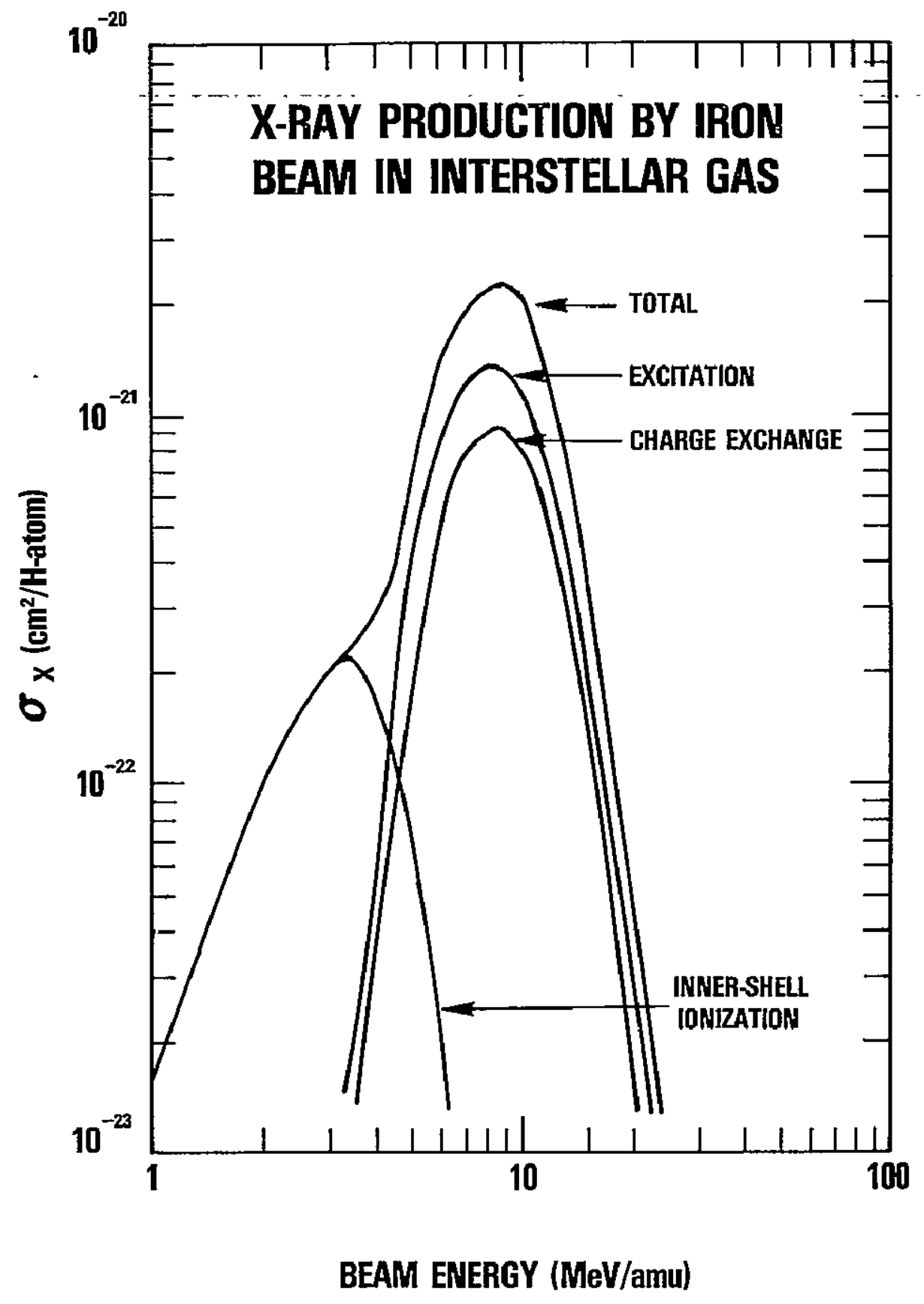

Figure 12 


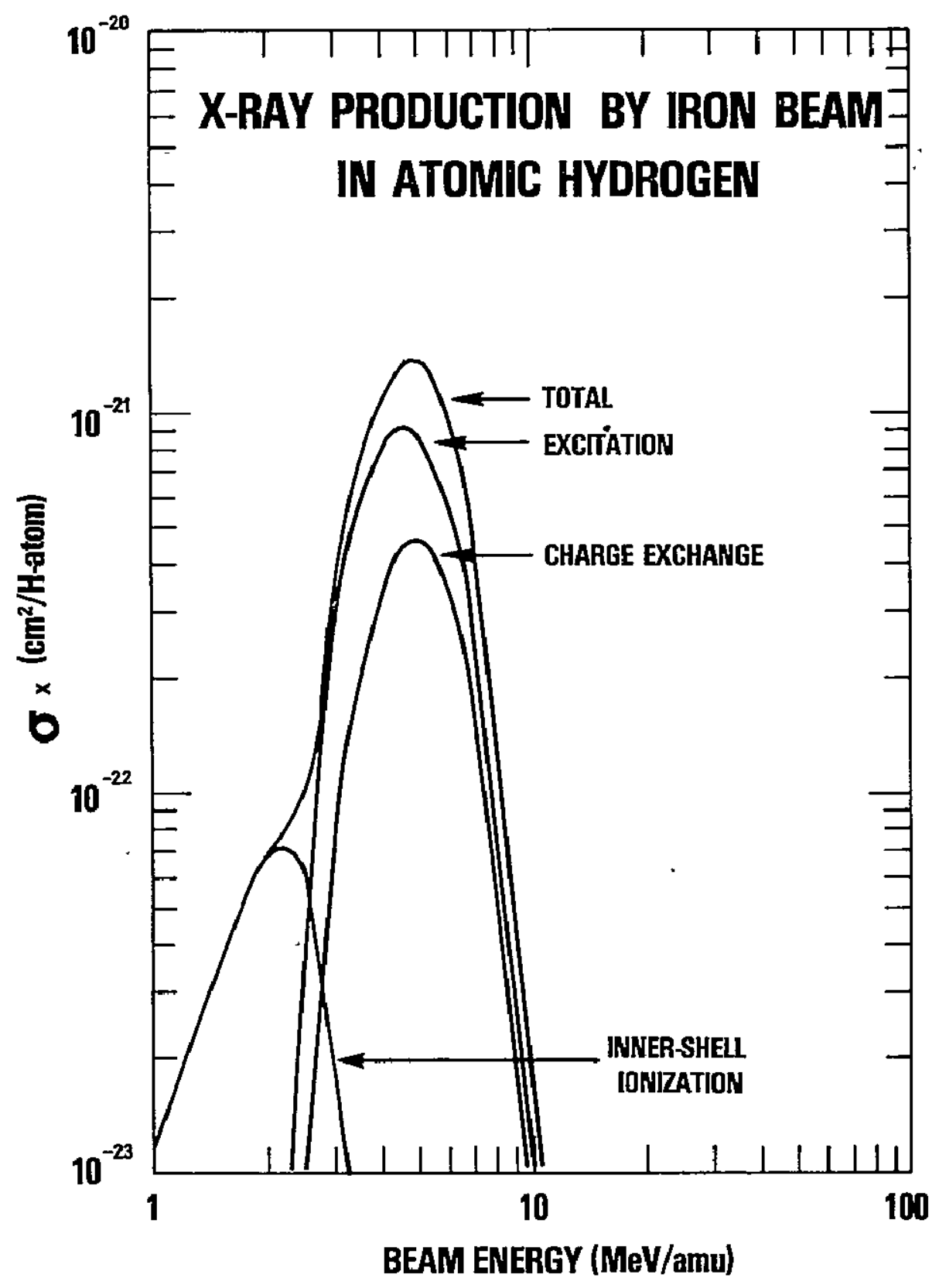

Figure 13 


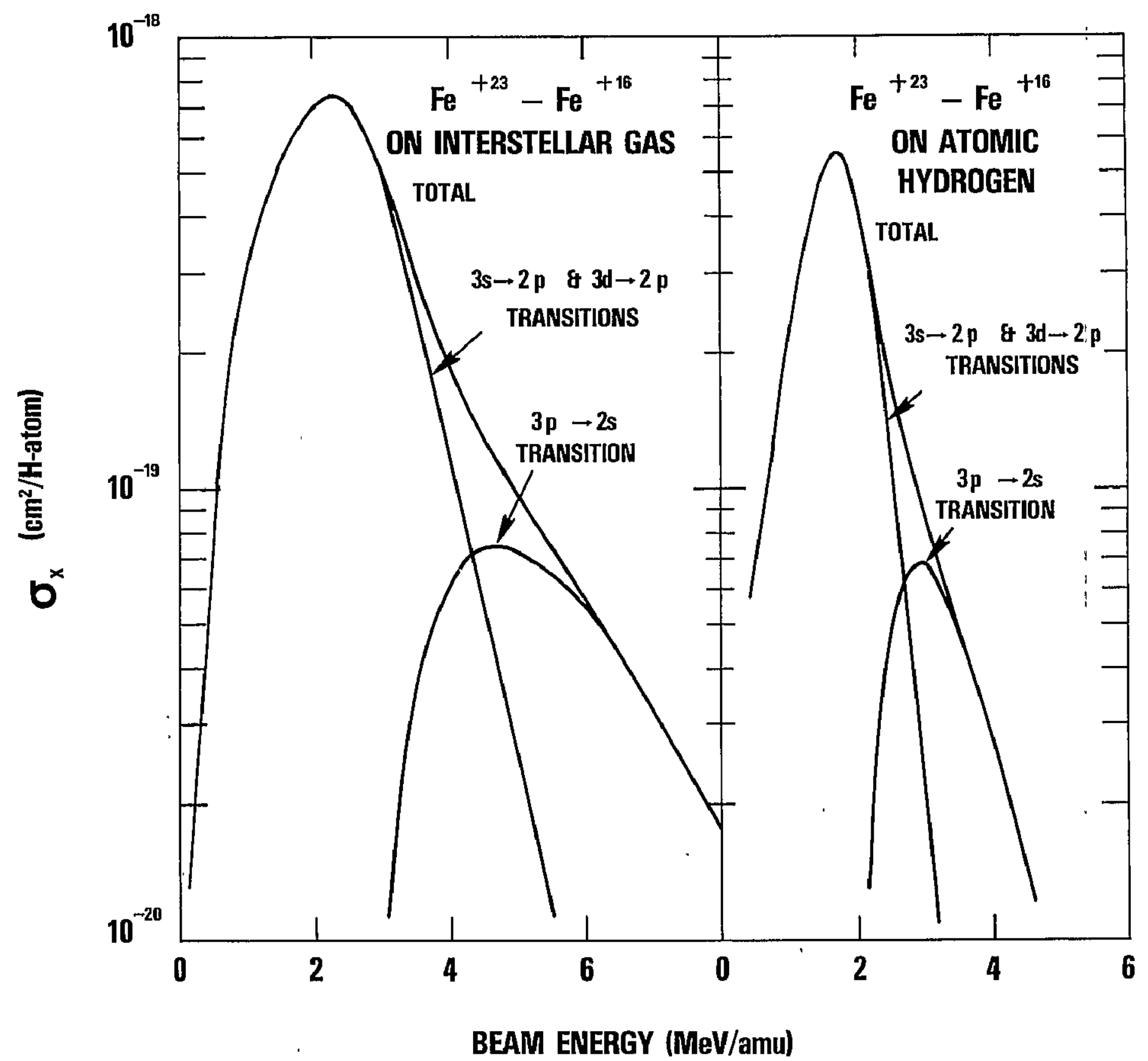

Figure 14 


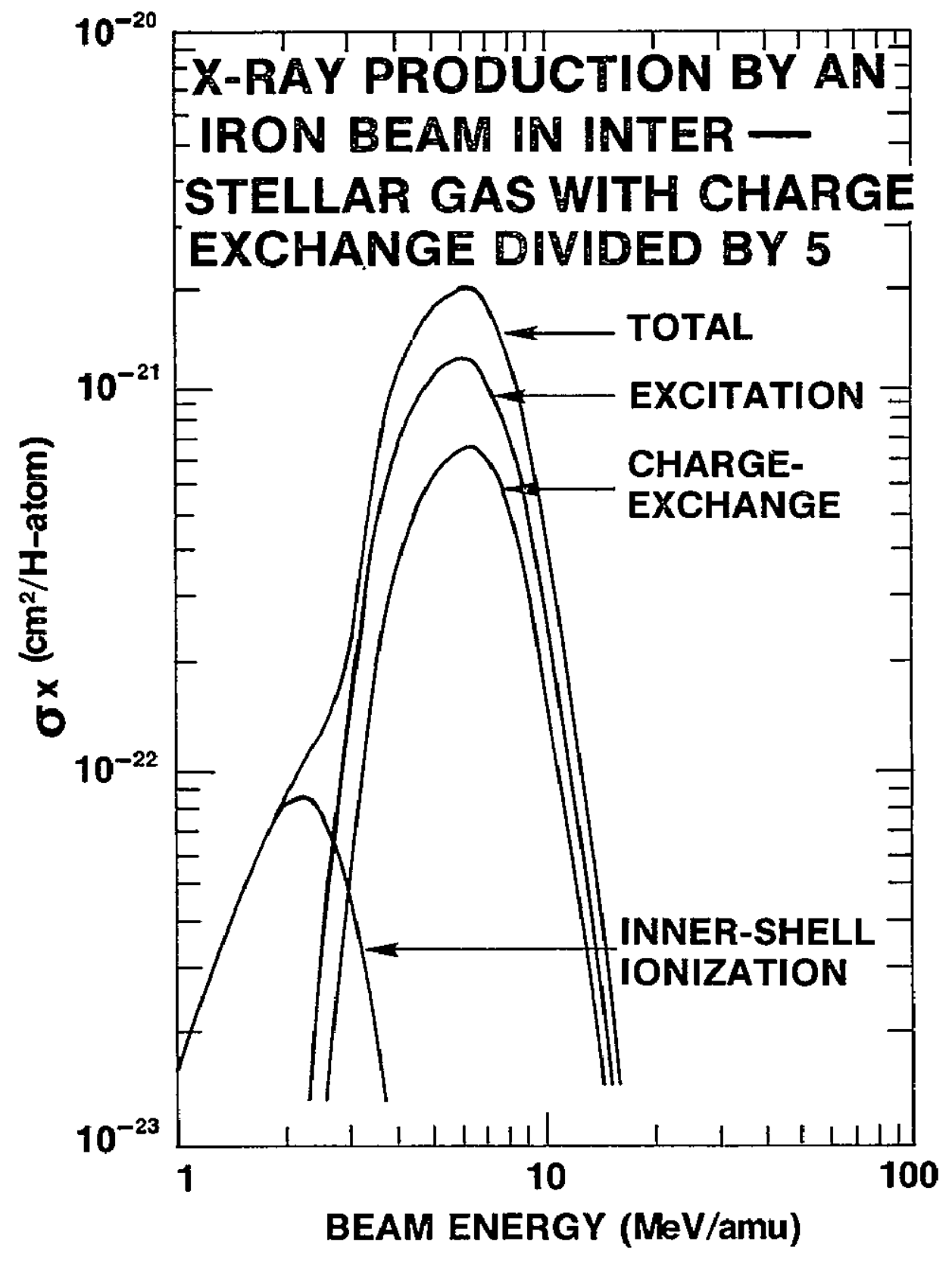

Figure 15 


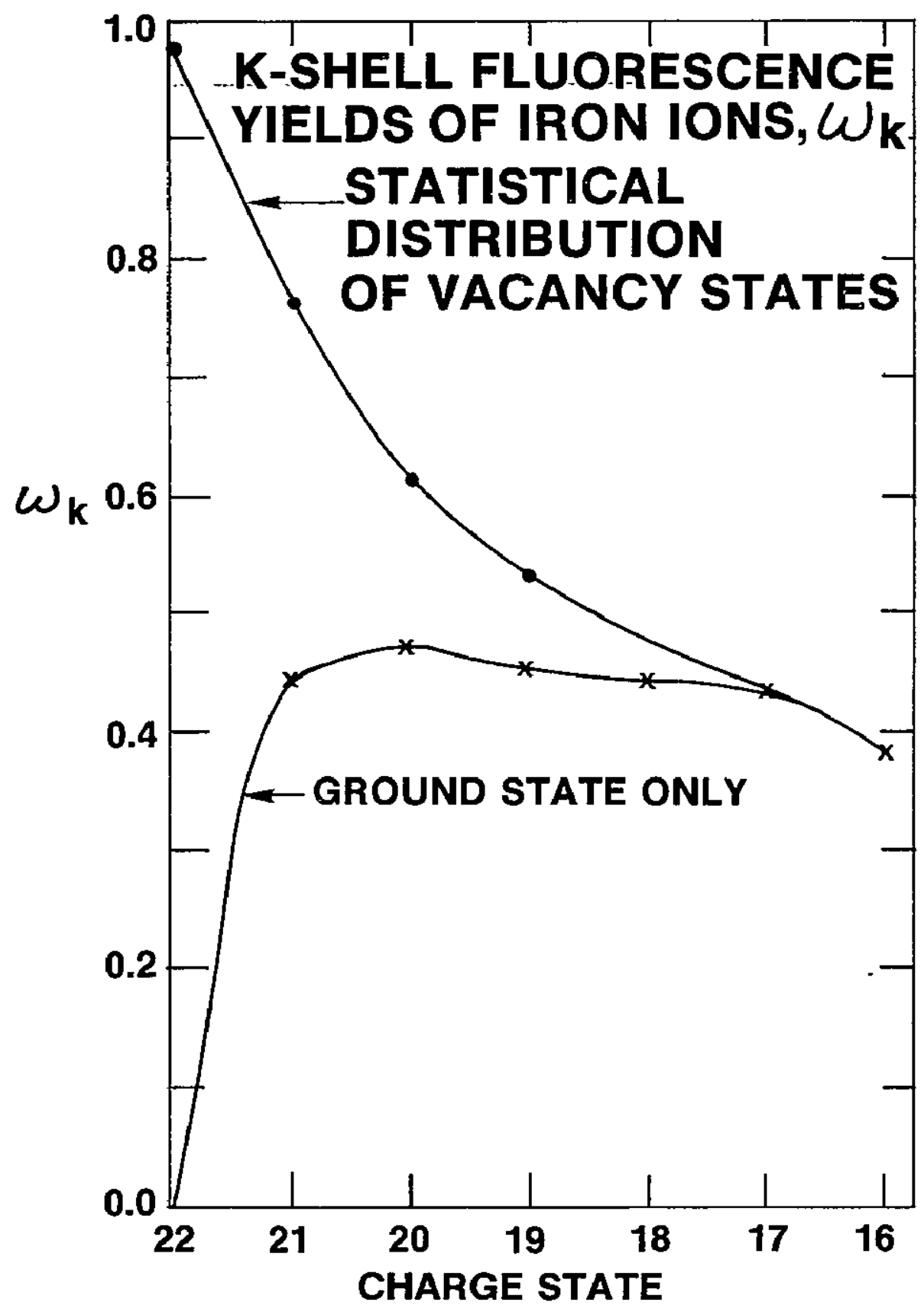

Figure 16 


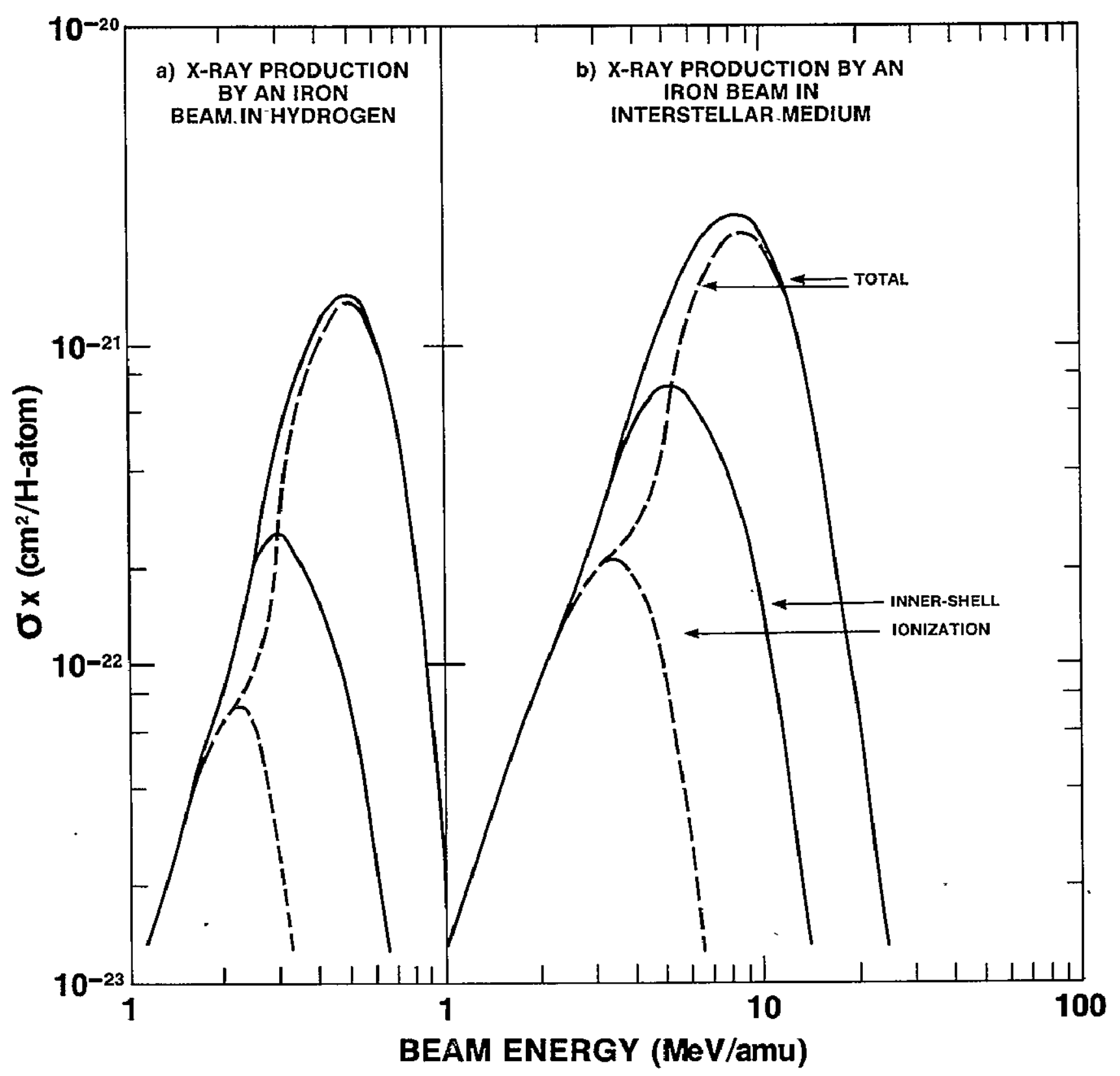

Figure 17 